\title{
Recoiling supermassive black holes in analytical and numerical galaxy potential
}

\author{
Majda Smole,${ }^{1 \star}$ Miroslav Micic, ${ }^{1}$ Ana Mitrašinović ${ }^{1}$ \\ 1 Astronomical Observatory, Volgina 7, 11060 Belgrade, Serbia
}

Accepted XXX. Received YYY; in original form ZZZ

\begin{abstract}
We follow trajectories of recoiling supermassive black holes (SMBHs) in analytical and numerical models of galaxy merger remnants with masses of $10^{11} \mathrm{M}_{\odot}$ and $10^{12} \mathrm{M}_{\odot}$. We construct various merger remnant galaxies in order to investigate how the central SMBH mass and the mass ratio of progenitor galaxies influence escape velocities of recoiling SMBHs. Our results show that static analytical models of major merger remnant galaxies overestimate the SMBHs escape velocities. During major mergers violent relaxation leads to the decrease of galaxy mass and lower potential at large remnant radii. This process is not depicted in static analytical potential but clearly seen in our numerical models. Thus, the evolving numerical model is a more realistic description of dynamical processes in galaxies with merging SMBHs. We find that SMBH escape velocities in numerical major merger remnant galaxies can be up to 25 per cent lower compared to those in analytical models. Consequently, SMBHs in numerical models generally reach greater galactocentric distances and spend more time on bound orbits outside of the galactic nuclei. Thus, numerical models predict a greater number of spatially-offset active galactic nuclei (AGNs).
\end{abstract}

Key words: black hole physics - gravitational waves - galaxies: interactions - methods: numerical

\section{INTRODUCTION}

According to the hierarchical galaxy formation, massive galaxies in local Universe have experienced numerous mergers. During the merger of two galaxies hosting SMBHs, due to the dynamical friction, SMBHs sink to the centre of the merger remnant, where they form a binary system. Further interactions with stars and gas carry away energy of the system which leads to the binary hardening.

In gas poor galaxies, SMBH binaries capture stars on centrophilic orbits and then eject them at higher velocities which results in decreasement of the separation between SMBHs (Begelman, Blandford \& Rees 1980; Ebisuzaki, Makino \& Okumura 1991). However, as binary system ejects stars, their central density decreases and once the loss cone is depleted, decay of SMBH binary is expected to stall at separation of $\lesssim 1$ pc, which is so-cold "final parsec problem" (e.g. Milosavljević \& Merritt 2001). SMBH binary coalescence timescale in spherical systems overcomes Hubble time and represents a bottleneck for SMBH mergers. On the other hand, numerical simulations have shown that SMBH coalescence timescales in triaxial and axisymmetric merger remnants are much shorter $(\sim 1 \mathrm{Gyr})$ and SMBH could be

^ E-mail: msmole@aob.rs, micic@aob.rs, amitrasinovic@aob.rs brought to separation $\ll 1$ pc before all stars on intersecting orbits have been ejected (e.g Berczik et al. 2006; Berentzen et al. 2009; Preto et al. 2011; Khan, Just \& Merritt 2011; Khan et al. 2013; Gualandris et al. 2017; Rantala et al. 2017). After gas rich galaxy merger, gas that is fueled to the central regions of galaxy may play important role in SMBH binary hardening. Interactions with massive gaseous nuclear disc and gas clumps can lead to the efficient SMBH coalescence within $10^{7}$ yr (Escala et al. 2004, 2005; Mayer et al. 2007; Fiacconi et al. 2013; Mayer 2013; Roškar et al. 2015; del Valle et al. 2015; Goicovic et al. 2016; Khan et al. 2016). However, in minor merger remnants SMBH sinking timescale depends on the orbital parameters and can be much longer (Callegari et al. 2009, 2011). Similar results have been found in the recent study by Tremmel et al. (2018) who used Romulus25 cosmological simulation to investigate timescales of SMBHs sinking down to subkpc scales in galaxies with different properties. Their results suggest that formation of close SMBH binary is strongly influenced by galaxy morphology and mass ratio of the progenitor galaxies, while some galaxy mergers do not necessary lead to SMBH merger.

Once the separation between SMBHs becomes $\lesssim 10^{-3}$ pc, gravitational wave radiation will efficiently extract angular momentum and energy from the binary system, causing 
rapid SMBHs merger (Begelman et al. 1980). Any asymmetry in the binary system, caused by SMBHs with unequal masses and/or spins, will lead to the asymmetric emission of gravitational radiation and SMBH recoil. Gravitational waves propagate in a preferential direction due to non-zero net linear momentum and the centre of mass of the binary recoils in the opposite direction (Redmount \& Rees 1989). In this process newly formed SMBH receives a kick, whose magnitude depends on the mass ratio of SMBHs, the spin magnitude and orientation with respect to the binary orbital plane, and the eccentricity of the orbit. With a breakthrough of the full numerical relativity simulations it has been shown that kick velocities can be as high as $\sim 4000 \mathrm{~km} \mathrm{~s}^{-1}$ (Gonzáles et al. 2007; Campanelli et al. 2007; Lousto \& Zlochower 2011), although super-kicks are considered to be rare and most SMBHs are ejected at lower velocities. Super-kicks are expected to occur for maximally spinning SMBHs, whose spin vectors prior to a merger are in the orbital plane and have opposite directions. However, in the presence of central gas disc torques from the disc can lead to SMBH spin alignment, and in that case even fast rotating SMBHs will not get kick velocities larger than $\sim 600 \mathrm{~km} \mathrm{~s}^{-1}$ (Blecha et al. 2016).

Super-kicks can eject SMBHs even from the massive elliptical galaxies, while lower kick velocities can be sufficient to remove SMBHs from the dwarf galaxies, globular clusters, and high redshift haloes, which generally have lower masses (Merritt et al. 2004; Micic, Abel \& Sigurdsson 2006; Volonteri 2007; Schnittman 2007 ; Sesana 2007; Volonteri, Gultekin \$ Dotti 2010; Micic, Holley-Bockelmann \& Sigurdsson 2011). Gravitational wave recoil can have negative influence on SMBH growth through mergers, since ejected SMBHs are less likely to merge with other SMBHs (e.g. Haiman 2004; Merritt et al. 2004; Volonteri 2007).

If a SMBH receives kick with amplitude lower than the escape velocity from the host, it may be displaced from the nucleus, and make several passages across the centre of the galaxy, before it sinks back to the core. Time scale for orbit decay depends on the kick velocity and oscillations can last up to several Gyr (Madau \& Quataert 2004; Gualandris \& Merritt 2008; Komossa \& Merritt 2008; Blecha \& Loeb 2008). Presence of the gas in the galactic centre can significantly influence recoiling SMBHs trajectories and in gas rich galaxies SMBHs will spend less time outside of the galactic nuclei (e.g. Blecha \& Loeb 2008; Devecchi et al. 2009; Blecha et al. 2011; Guedes et al. 2011; Sijacki, Springel \& Haehnelt 2011).

Effects of the host halo accretion on the recoiling SMBH trajectories have been studied analytically by Smole (2015) and Choksi et al. (2017). Both works have shown that host halo growth significantly influences SMBH escape velocities since SMBHs can be easily ejected from low mass galaxies at high redshifts, while halo accretion at later times makes the ejection harder.

Recoiling SMBHs could be observed as spatially or kinematically offset AGNs (e.g. Madau \& Quataert 2004; Loeb 2007; Blecha \& Loeb 2008; Blecha et al. 2011; Sijacki, Springel \& Haehnelt 2011). Spatially-offset AGNs are characterized by an accreting SMBH displaced from the host galaxy centre. In the case of kinematically-offset AGN broad line region is ejected together with the $\mathrm{SMBH}$, while narrow line region stays associated with the galaxy nucleus. Hence, ejection of the SMBH produces a difference between velocities of these two regions. Numerous candidate offset AGNs have been observed, although alternative explanations cannot be ruled out (we refer to Blecha et al. 2016 for more detailed summary of offset AGN observations).

In order to test the observability of the offset AGN, Blecha et al. (2016) investigated trajectories of recoiling SMBHs using Illustris cosmological simulations (Vogelsberger et al. 2014a, 2014b). By extracting properties of merging SMBHs and progenitor galaxies directly from the simulation, authors constructed analytical models of merger remnants galaxies consisted of dark matter halo (DMH), bulge and circumnuclear disc. Further, they assumed different SMBH spin distributions to estimate resulting kick velocities which are then assigned to central SMBHs whose trajectories are integrated.

In this paper, we apply similar analytical model as described in Blecha et al. (2016) to construct merger remnant galaxies of specific masses. Instead of using cosmological simulation to extract properties of remnant galaxies, in our model merger remnant galaxy is determined by its total mass, the central SMBH mass and the scaling relations. In addition to this, we construct numerical models of the merging galaxies in order to obtain remnants with characteristics similar to those in analytical models. We calculate escape velocities of SMBHs ejected from the galaxies which have been formed in major or minor mergers. The capability of different hosts to retain recoiling SMBHs is explored with special focus on differences between analytical and numerical models of galaxies. In Section 2 we describe the method. In Section 3 we present our results. We summarize and discuss our results in Section 4.

\section{METHODS}

We follow trajectories of the recoiling SMBHs in analytical and numerical galaxy potential whose components are DMH, bulge and disc. Total masses of the considered galaxies are $10^{11} \mathrm{M}_{\odot}$ and $10^{12} \mathrm{M}_{\odot}$. The chosen masses are of the greatest significance for studying recoiling SMBHs. More massive galaxies have deep gravitational well and only rare super-kicks could remove SMBHs from their hosts. In less massive galaxies, kick velocities $\lesssim 100 \mathrm{~km} \mathrm{~s}^{-1}$ could lead to the complete SMBH ejection. Furthermore, the estimated distribution of recoil kick velocities suggests that kick amplitudes in interval $100 \lesssim v_{\text {kick }} \lesssim 1000 \mathrm{~km} \mathrm{~s}^{-1}$ are the most common kicks (Blecha et al. 2016). In order to investigate if $\mathrm{SMBH}$ trajectories are sensitive to the mass ratio of the progenitor galaxies, we separately model major (1:1) and minor (1:10) mergers. Assuming that central SMBHs in major merger progenitors have comparable masses, the only source of asymmetry in the SMBH binary system are SMBH spins. Depending on the spin amplitude and the orientation, SMBH kick velocities in major merger remnants could range from few tens up to $\sim 2000 \mathrm{~km} \mathrm{~s}^{-1}$. On the other hand, unequal mass SMBHs in 1:10 mergers have narrower interval of possible kick velocities, $50 \lesssim v_{\text {kick }} \lesssim 500 \mathrm{~km} \mathrm{~s}^{-1}$ (Micic et al. 2011).

Since dynamical friction force increases with the recoiling SMBH mass, we consider two different galaxy models. In the first model, merger remnant galaxy hosts central 
SMBH with mass $M_{\mathrm{BH}}=M_{\text {gal }} / 10^{5}$, which is the expected SMBH mass according to $M_{\mathrm{BH}}-M_{\text {halo }}$ relation (Ferrarese 2002). In further text we address this model as galaxies with $M_{\mathrm{BH}}-M_{\text {halo }}$ central $\mathrm{SMBH}$. In the second model, $\mathrm{SMBH}$ mass is $M_{\mathrm{BH}}=M_{\mathrm{gal}} / 10^{3}$, which is the maximal expected $\mathrm{SMBH}$ mass derived from the following argument: if mass in baryons is approximately 16 per cent of the dark matter (DM) mass and if a typical SMBH mass to bulge mass ratio is 0.5 per cent (Kormendy \& Ho 2013), then $\sim 8 \times 10^{-4}$ of the halo mass is the gas that SMBH can accrete. This model will be addressed as galaxies with over-massive central SMBH. Since scaling relations suggest that central SMBH mass and properties of the host galaxies are tightly coupled, an additional motivation to compare escape velocities from galaxies with different central SMBH masses arises. With these two models we are able to follow SMBH ejections in galaxies with typical SMBH masses and also in galaxies with unusually massive central SMBH. More detailed description of our models is given in Section 2.1. Parameters of our galaxy models are given in Table 1 and Table 2.

For each of the galaxy models described above we use the following procedure: 1) generate initial conditions for numerical pre-merger galaxy, 2) evolve galaxy in isolation in order to test stability of each galaxy component, 3) produce the exact analytical counterpart, 4) test the accuracy of our numerical method by comparing, mass profiles, escape velocities and recoiling SMBH trajectories in numerical and analytical pre-merger galaxies, 5) simulate galaxy merger, 6) compare SMBH trajectories in numerical merger remnants to those in analytical models of isolated galaxies. Initial conditions for numerical models are generated using Galact ICS code (Kuijken \& Dubinski 1995; Widrow \& Dubinski 2005; Widrow, Pym \& Dubinski 2008), while galaxy evolution, mergers and trajectories of kicked SMBHs are simulated using GADGET-2 code (Springel 2005). Our main goal is to test how redistribution of mass within post-merger galaxy affects its capability to retain a recoiling SMBH. We describe each of these steps in more details bellow.

\subsection{Progenitor galaxy models}

\subsubsection{Numerical models}

Initial conditions for numerical models are generated using GalactICS code (Kuijken \& Dubinski 1995; Widrow \& Dubinski 2005; Widrow, Pym \& Dubinski 2008). For the given SMBH mass and the total galaxy mass, masses of individual galaxy components are calculated from scaling relations. Bulge mass is derived using $M_{\mathrm{bulge}}-M_{\mathrm{BH}}$ relation (Marconi \& Hunt 2003), while disc mass is estimated using $M_{\mathrm{DM}}-M_{\text {gal,b }}$ relation (Moster, Naab \& White 2013). $M_{\text {gal,b }}$ represents total galaxy mass in baryons, i.e. total disc and bulge mass, thus the disc mass can be calculated subtracting the bulge mass from the total mass in baryons, estimated from the relation. $M_{\text {bulge }}-M_{\mathrm{BH}}$ relation predicts that galaxies that harbor over-massive central SMBHs also have massive bulges, so the total baryonic component of the galaxy estimated using $M_{\mathrm{DM}}-M_{\mathrm{gal}, \mathrm{b}}$ relation is in the form of bulge, and in those models disc component is neglected.

Initial conditions for gas particles are calculated from the previously generated N-body models, following the procedure given by Gajda, Lokas \& Athanassoula (2018). We randomly select 20 per cent of disc particles and convert them to gas particles with initial temperature set to $2000 \mathrm{~K}$. Gas particle velocities are calculated as the tangential velocities at the galaxy mid-plane, while radial and vertical velocities are set to zero. Gas component is only included in simulations of galaxies whose central SMBH has mass of $M_{\mathrm{BH}}=M_{\text {gal }} / 10^{5}$. Galaxies with over-massive SMBHs do not have disc or gas components and we will refer to those galaxies as elliptical.

Each progenitor galaxy is represented with $N=10^{6}$ particles. While distribution of particles between galaxy components, as well as particle masses, depends on the galaxy model. For instance, particle distribution in major merger progenitor of $10^{12} \mathrm{M}_{\odot}$ galaxy which harbors $M_{\mathrm{BH}}-M_{\text {halo }} \mathrm{SMBH}$ is $N_{\text {halo }} \sim 5 \times 10^{5}, N_{\text {disc }} \sim 3.6 \times 10^{5}$, $N_{\text {bulge }} \sim 5 \times 10^{4}$ and $N_{\text {gas }} \sim 9 \times 10^{4}$, while major progenitor of galaxy with over-massive central SMBH has different distribution, $N_{\text {halo }} \sim 6 \times 10^{5}$ and $N_{\text {bulge }} \sim 4 \times 10^{5}$. With this mass resolution, SMBH mass is always at least one order of magnitude larger than a DM particle mass, i.e. two orders of magnitude larger than a stellar or gaseous particle mass. Since we are only interested in general interaction between ejected SMBH and the host galaxy, this mass ratio is sufficient to track the dynamic of the recoiling SMBH. SMBH is represented as one particle, placed at the centre of mass calculated with respect to the most gravitationally bound 5 per cent bulge particles.

A fixed value for gravitational softening length parameter, $\epsilon=0.1 \mathrm{kpc}$, is used in all simulations and for all particle types. Value of the softening length parameter scales with the number of particles in the system and with dimension of the system as $R / N^{1 / 3}<\epsilon<R / N^{1 / 2}$ (Binney \& Tremaine 2008). In practice, several criteria on the selection of the optimal softening length have been proposed (e.g. Merritt 1996; Dehnen 2001; Power et al. 2003; Zhang et al. 2019). Choosing $\epsilon_{\text {opt }} \simeq 2 R / N^{1 / 2}$ as an optimal softening length (Zhang et al. 2019) yields values in range $0.2 \mathrm{kpc} \lesssim \epsilon_{\mathrm{opt}} \lesssim 0.5 \mathrm{kpc}$ for DM particles in different models, and $\epsilon_{\text {opt }} \lesssim 0.1 \mathrm{kpc}$ for baryonic particles. However, using the same softening length for all particle types significantly reduces computational time. In order to validate the usage of a smaller rather than optimal value of the softening length for DM particles, we run test simulations adjusting $\epsilon_{\mathrm{DM}}=0.5 \mathrm{kpc}$ for DM particles and $\epsilon_{\mathrm{b}}=0.1 \mathrm{kpc}$ for baryonic particles. Test runs did not show any differences in galaxy mass profiles over 3 Gyr compared to the runs with a fixed value of gravitational softening length, $\epsilon=0.1 \mathrm{kpc}$. Additionally, difference between SMBH escape velocity, which is calculated over $10 \mathrm{Gyr}$, is bellow 1 per cent.

After generating initial conditions for numerical galaxy models, we test their stability. Evolution of each progenitor galaxy is simulated in isolation, so that eventual changes in galaxy profiles that emerge due to galaxy model itself can be distinguished from galaxy interactions. Galaxy is assumed to be stable if profiles of galaxy components do not significantly vary with time over 3 Gyr. Fig. 1 shows an example of stability test run for the progenitor of the minor merger galaxy with mass $10^{12} \mathrm{M}_{\odot}$ and SMBH mass of $10^{7} \mathrm{M}_{\odot}$. Results of stability tests for other progenitor galaxies are given in Appendix A. 


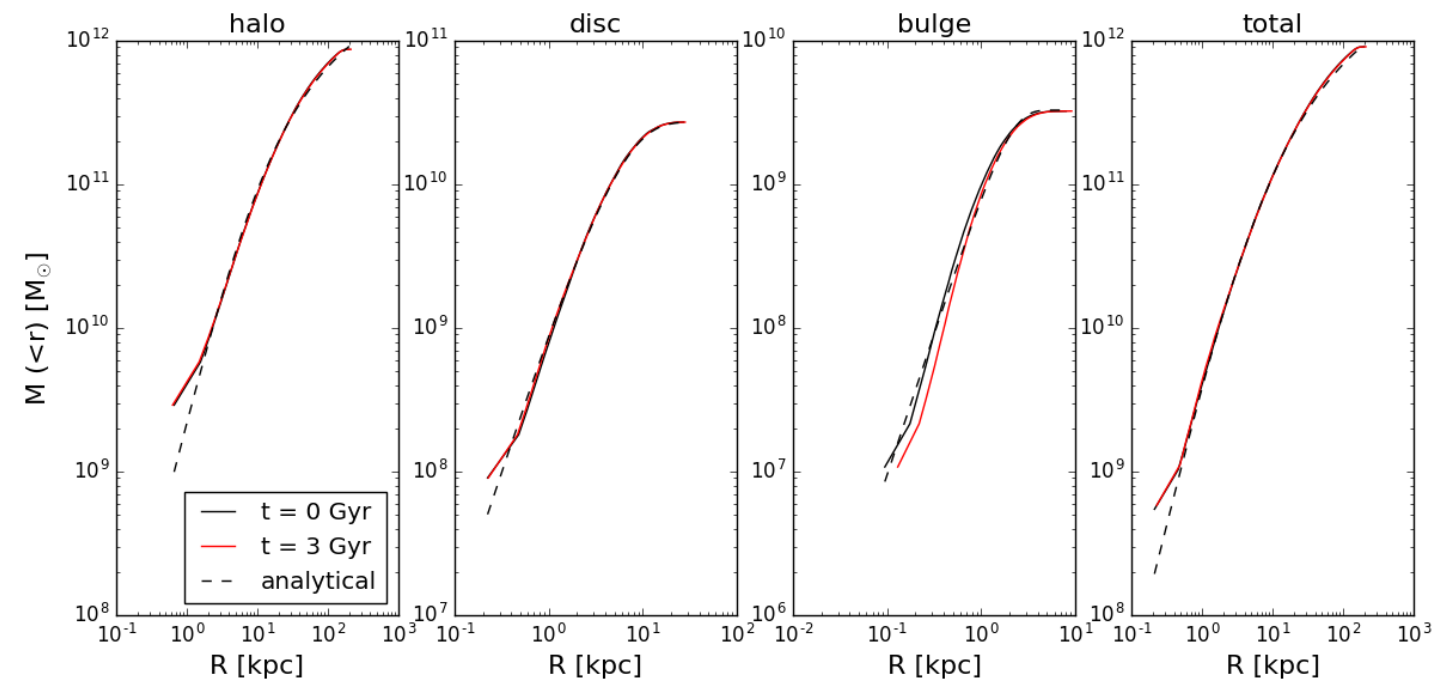

Figure 1. Mass profiles in analytical (dashed lines) and numerical (solid lines) galaxy models. For numerical models mass profiles of individual galaxy components are shown at the beginning of the simulation and 3 Gyr later.

Table 1. Parameters of progenitor galaxies.

\begin{tabular}{|c|c|c|c|c|c|c|c|c|c|c|c|}
\hline $\begin{array}{l}M_{\text {gal }} \\
{\left[\mathrm{M}_{\odot}\right]}\end{array}$ & $\begin{array}{l}M_{\mathrm{BH}} \\
{\left[\mathrm{M}_{\odot}\right]}\end{array}$ & $\begin{array}{l}\text { merger } \\
\text { ratio }\end{array}$ & $\begin{array}{l}\text { progenitor } \\
\text { galaxy }\end{array}$ & $\begin{array}{l}M_{\text {halo }} \\
{\left[\mathrm{M}_{\odot}\right]}\end{array}$ & $\begin{array}{l}r_{\mathrm{s}} \\
{[\mathrm{kpc}]}\end{array}$ & $c$ & $\begin{array}{l}M_{\text {bulge }} \\
{\left[\mathrm{M}_{\odot}\right]}\end{array}$ & $\begin{array}{l}r_{\text {bulge }} \\
{[\mathrm{kpc}]}\end{array}$ & $\alpha$ & $\begin{array}{l}M_{\text {disc }} \\
{\left[\mathrm{M}_{\odot}\right]}\end{array}$ & $\begin{array}{l}R_{\text {disc }} \\
{[\mathrm{kpc}]}\end{array}$ \\
\hline \multirow{6}{*}{$1 \cdot 10^{12}$} & \multirow{3}{*}{$1 \cdot 10^{7}$} & $1: 1$ & \multirow{4}{*}{$\begin{array}{l}\text { primary } \\
\text { secondary }\end{array}$} & $4.77 \cdot 10^{11}$ & 10.42 & 15.80 & $1.73 \cdot 10^{9}$ & 1.90 & 1.02 & $1.58 \cdot 10^{10}$ & 3.70 \\
\hline & & $1: 10$ & & $8.72 \cdot 10^{11}$ & 9.20 & 19.50 & $3.24 \cdot 10^{9}$ & 1.95 & 1.03 & $3.39 \cdot 10^{10}$ & 3.60 \\
\hline & & & & $8.91 \cdot 10^{10}$ & 10.50 & 10.30 & $3.25 \cdot 10^{8}$ & 1.72 & 1.13 & $3.49 \cdot 10^{9}$ & 2.40 \\
\hline & \multirow{3}{*}{$1 \cdot 10^{9}$} & $1: 1$ & & $2.71 \cdot 10^{11}$ & 11.10 & 11.73 & $1.52 \cdot 10^{11}$ & 18.70 & 1.21 & $T$ & $T$ \\
\hline & & $1: 10$ & primary & $4.97 \cdot 10^{11}$ & 12.20 & 11.50 & $4.03 \cdot 10^{11}$ & 19.50 & 1.20 & 1 & 1 \\
\hline & & & secondary & $5.84 \cdot 10^{10}$ & 10.80 & 9.00 & $4.18 \cdot 10^{10}$ & 11.00 & 1.20 & / & / \\
\hline \multirow{6}{*}{$1 \cdot 10^{11}$} & \multirow{3}{*}{$1 \cdot 10^{6}$} & $1: 1$ & \multirow{3}{*}{$\begin{array}{l}\text { primary } \\
\text { secondary }\end{array}$} & $4.93 \cdot 10^{10}$ & 10.90 & 8.00 & $1.73 \cdot 10^{8}$ & 1.50 & 1.02 & $3.80 \cdot 10^{8}$ & 2.10 \\
\hline & & $1: 10$ & & $8.94 \cdot 10^{10}$ & 10.70 & 10.50 & $3.03 \cdot 10^{8}$ & 1.60 & 1.02 & $6.79 \cdot 10^{8}$ & 2.50 \\
\hline & & & & $8.99 \cdot 10^{9}$ & 3.02 & 11.02 & $3.03 \cdot 10^{7}$ & 0.26 & 0.80 & $8.64 \cdot 10^{7}$ & 2.30 \\
\hline & \multirow{3}{*}{$1 \cdot 10^{8}$} & $1: 1$ & \multirow{3}{*}{$\begin{array}{l}\text { primary } \\
\text { secondary }\end{array}$} & $2.90 \cdot 10^{10}$ & 11.00 & 6.90 & $2.04 \cdot 10^{10}$ & 4.60 & 1.20 & 1 & 1 \\
\hline & & $1: 10$ & & $5.32 \cdot 10^{10}$ & 7.58 & 8.80 & $3.70 \cdot 10^{10}$ & 7.40 & 1.21 & / & / \\
\hline & & & & $5.43 \cdot 10^{9}$ & 3.00 & 11.50 & $3.70 \cdot 10^{9}$ & 4.10 & 1.20 & / & / \\
\hline
\end{tabular}

\subsubsection{Analytical models}

Further, we make analytical models of progenitor galaxies with the same characteristics as their numerical counterparts. Density profiles of each of the galaxy components are described bellow and profile parameters are given in Table 1.

$\mathrm{DMH}$ is described by NFW density profile (Navarro, Frenk \& White 1997):

$\rho_{\text {halo }}(r)=\frac{\rho_{\text {halo }, 0}}{\frac{r}{r_{\mathrm{s}}}\left(1+\frac{r}{r_{\mathrm{s}}}\right)^{2}}$,

where $r_{\mathrm{s}}$ and $\rho_{\text {halo, } 0}$ are the scale radius and the characteristic density, respectively.

Bulge component is modeled as a power-law density spherical potential with an exponential cut-off (Bovy \& Rix 2013):

$\rho_{\text {bulge }}(r)=\rho_{\text {bulge }, 0}\left(\frac{r_{\text {bulge }}}{r}\right)^{\alpha} \times \exp \left[-\left(r / r_{\text {bulge }}\right)^{2}\right]$,

where $r_{\text {bulge }}$ and $\rho_{\text {bulge }, 0}$ represent characteristic bulge radius and density, respectively.
Disc mass distribution is represented as an exponential disc with a surface density:

$\Sigma_{\text {disc }}(R)=\Sigma_{\text {disc }, 0} \times \exp \left(-R / R_{\text {disc }}\right)$.

where $\Sigma_{\text {disc }, 0}$ is the central surface density, $R_{\text {disc }}$ is disc scale length and $R$ is the distance from the galaxy centre in the disc plane. As discussed above, disc component is not included in models with over-massive SMBHs, since in those models total baryonic component is in the form of massive bulge.

Table 1 summarize parameters of progenitor galaxies used in analytical models. Those parameters represent a fit to the mass profiles of galaxy components resulting from numerical models. An example of mass profiles of analytical and numerical galaxies is shown on Fig 1. Comparison of mass profiles in analytical and numerical models for all progenitor galaxies is shown in Appendix A, together with the stability test of numerical models. 


\section{$2.2 \quad$ SMBH trajectories}

\subsubsection{Analytical models}

Next step is to integrate the trajectory of recoiling SMBH under the influence of potential described above. SMBH at a position $\mathbf{x}$ experiences gravitational force generated by a distribution of mass $\rho\left(\mathbf{x}^{\prime}\right)$ (Binney \& Tremaine 2008):

$\mathbf{F}(\mathbf{x})=M_{\mathrm{BH}} \mathbf{g}(\mathbf{x})$,

where $\mathbf{g}(\mathbf{x})=G \int \mathrm{d}^{3} \mathbf{x}^{\prime} \frac{\mathbf{x}^{\prime}-\mathbf{x}}{\left|\mathbf{x}^{\prime}-\mathbf{x}\right|^{3}} \rho\left(\mathbf{x}^{\prime}\right)$ is gravitational field. If the gravitational potential is defined as:

$\Phi(\mathbf{x}) \equiv-G \int \mathrm{d}^{3} \mathbf{x}^{\prime} \frac{\rho\left(\mathbf{x}^{\prime}\right)}{\left|\mathbf{x}^{\prime}-\mathbf{x}\right|}$,

gravitational field can be calculated as:

$\mathbf{g}(\mathbf{x})=-\nabla_{\mathbf{x}} \Phi$.

Here, $\Phi$ is the total gravitational potential from all galaxy components. However, potential of an exponential disc cannot be calculated analytically. We adopt a toy model described by Geehan et al. (2006) where disc mass is spherically distributed and whose potential is then:

$\Phi_{d}(r)=-2 \pi G \Sigma_{\mathrm{d}, 0} R_{\mathrm{d}}^{2}\left(\frac{1-e^{r / R_{\mathrm{d}}}}{r}\right)$.

The authors have demonstrated that rotation curve for disc component shows only minor changes when calculated for spherical and axisymmetric case (figure 2, Geehan et al. 2006).

In addition to gravitational force, SMBH also experiences dynamical friction force against stars and DM. We include both stellar and DM dynamical friction via the Chandrasekhar formula (Chandrasekhar 1943). Assuming a Maxwellian velocity distribution, a SMBH moving with velocity $v_{\mathrm{BH}}$ through a background density $\rho_{\text {bulge }}$ or $\rho_{\text {halo }}$ will experience dynamical friction force:

$\mathbf{f}_{\mathrm{df}}=-I(\mathcal{M}) \times \frac{4 \pi \rho\left(G M_{\mathrm{BH}}\right)^{2}}{\sigma^{2}} \frac{\boldsymbol{v}_{\mathrm{BH}}}{v_{\mathrm{BH}}}$,

with

$I(\mathcal{M})=\frac{\ln (\Lambda)}{\mathcal{M}^{2}}\left(\operatorname{erf}\left(\frac{\mathcal{M}}{\sqrt{2}}\right)-\sqrt{\frac{2}{\pi}} \mathcal{M} e^{-\mathcal{M}^{2} / 2}\right)$,

where $\mathcal{M} \equiv v_{\mathrm{BH}} / \sigma$ is the Mach number and Coulomb logarithm is $\ln (\Lambda)=3.1$ (Blecha \& Loeb 2008). Velocity dispersion for stellar component can be calculated using $M_{\mathrm{BH}}-\sigma_{\text {bulge }}$ (McConnell \& Ma 2013) relation. Following Tanaka \& Haiman (2009) and Choksi et al. (2017) we use simplified model and assume constant DM velocity dispersion. Velocity dispersion is independent of the radius for an isothermal sphere. Several studies show a tight correlation between stellar and DM velocity dispersions, suggesting nearly isothermal total density profiles of quiescent galaxies (Schechter 2016, Zahid et al. 2016, Zahid, Sohn \& Geller 2018). Here we adopt a relation between DMH mass and DM velocity dispersion ( $\left.M_{\text {halo }}-\sigma_{\mathrm{DM}}\right)$ derived using Illustris cosmological simulation (Zahid et al. 2018).

Trajectory of the recoiling SMBH is governed by the equation of motion in the form:

$\ddot{x}=\left(-\nabla_{\mathbf{x}} \Phi+\boldsymbol{a}_{\mathrm{df}, \mathrm{DM}}+\boldsymbol{a}_{\mathrm{df}, \text { bulge }}\right) \hat{\mathbf{x}}$.

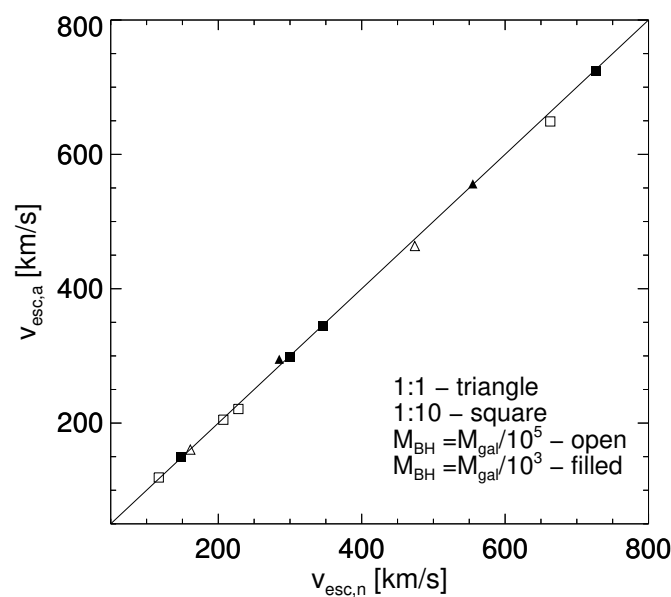

Figure 2. Escape velocities from progenitor galaxies in analytical models as a function of their escape velocities in numerical models, for galaxies with different central SMBH masses (open and filled symbols). Major merger remnants are represented with triangles and minor merger remnants with squares.

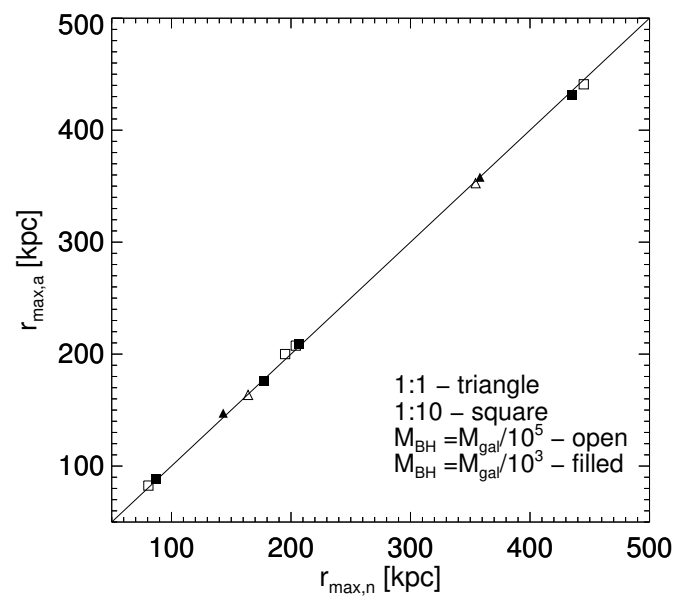

Figure 3. Maximal separation of a recoiling $\mathrm{SMBH}$ from the galaxy centre over a Hubble time in analytical models, as a function of $r_{\max }$ in numerical models. SMBH kick velocity is equal to the escape velocity for each galaxy model. Notations are the same as in Fig. 2 .

SMBH is initially placed at the galaxy centre with an assigned kick velocity in orthogonal direction with respect to the disc plane. SMBH trajectory is numerically integrated with a time-step $\Delta t=10^{4} \mathrm{yr}$, using leapfrog integration.

We assign various kick velocities to the SMBHs in our models and follow their trajectories until the recoiling SMBH returns to the galaxy halo or until integration reaches a Hubble time. Galaxy escape velocity is defined as a SMBH kick velocity necessary for a SMBH to return to its host halo after $\gtrsim 10$ Gyr. 
Table 2. Parameters of analytical merger remnant galaxies.

\begin{tabular}{|c|c|c|c|c|c|c|c|c|c|c|}
\hline $\begin{array}{l}M_{\text {gal }} \\
{\left[\mathrm{M}_{\odot}\right]}\end{array}$ & $\begin{array}{l}M_{\mathrm{BH}} \\
{\left[\mathrm{M}_{\odot}\right]}\end{array}$ & $\begin{array}{l}\text { merger } \\
\text { ratio }\end{array}$ & $\begin{array}{l}M_{\text {halo }} \\
{\left[\mathrm{M}_{\odot}\right]}\end{array}$ & $\begin{array}{l}r_{\mathrm{s}} \\
{[\mathrm{kpc}]}\end{array}$ & $c$ & $\begin{array}{l}M_{\text {bulge }} \\
{\left[\mathrm{M}_{\odot}\right]}\end{array}$ & $\begin{array}{l}r_{\mathrm{b}} \\
{[\mathrm{kpc}]}\end{array}$ & $\alpha$ & $\begin{array}{l}M_{\text {disc }} \\
{\left[\mathrm{M}_{\odot}\right]}\end{array}$ & $\begin{array}{l}r_{\mathrm{s}} \\
{[\mathrm{kpc}]}\end{array}$ \\
\hline \multirow{3}{*}{$1 \cdot 10^{12}$} & $1 \cdot 10^{7}$ & $1: 1$ & $9.54 \cdot 10^{11}$ & 10.70 & 17.90 & $3.51 \cdot 10^{10}$ & 6.90 & 1.13 & / & / \\
\hline & & $1: 10$ & $9.59 \cdot 10^{11}$ & 9.50 & 19.50 & $3.57 \cdot 10^{9}$ & 2.10 & 0.90 & $3.80 \cdot 10^{10}$ & 3.80 \\
\hline & $1 \cdot 10^{9}$ & $1: 1$ & $5.44 \cdot 10^{11}$ & 11.18 & 13.04 & $4.44 \cdot 10^{11}$ & 18.20 & 1.10 & / & T \\
\hline \multirow{5}{*}{$1 \cdot 10^{12}$} & & $1: 10$ & $5.53 \cdot 10^{11}$ & 11.40 & 14.00 & $4.41 \cdot 10^{11}$ & 19.50 & 1.20 & / & / \\
\hline & $1 \cdot 10^{6}$ & $1: 1$ & $9.86 \cdot 10^{10}$ & 10.10 & 10.30 & $1.32 \cdot 10^{9}$ & 3.80 & 1.11 & 1 & 1 \\
\hline & & $1: 10$ & $9.81 \cdot 10^{10}$ & 10.30 & 10.10 & $3.35 \cdot 10^{8}$ & 1.50 & 0.90 & $9.48 \cdot 10^{8}$ & 2.50 \\
\hline & $1 \cdot 10^{8}$ & $1: 1$ & $5.45 \cdot 10^{10}$ & 7.50 & 8.60 & $4.45 \cdot 10^{10}$ & 7.80 & 1.10 & / & 1 \\
\hline & & $1: 10$ & $5.86 \cdot 10^{10}$ & 7.58 & 8.60 & $4.13 \cdot 10^{10}$ & 7.40 & 1.21 & / & / \\
\hline
\end{tabular}

\subsubsection{Numerical models}

In the numerical models, SMBH is represented as one massive particle placed at the galaxy centre. Initial SMBH position is calculated with respect to the most gravitationally bound 5 per cent bulge particles. As in the analytical models, galaxy escape velocity is defined as a SMBH kick velocity necessary for a SMBH to return to its host halo after $\gtrsim 10$ Gyr.

In order to validate the accuracy of numerical method, we compare escape velocities from analytical and numerical models of progenitor galaxies, as well as the maximal separation from the galaxy centre a $\mathrm{SMBH}$ can reach over 10 Gyr. Results are shown in Fig. 2 and Fig. 3 for all progenitors of $10^{11} \mathrm{M}_{\odot}$ and $10^{12} \mathrm{M}_{\odot}$ galaxies. Escape velocities and maximal separations of SMBHs in analytical and numerical models are in good agreement for all pre-merger galaxies.

\subsection{Galaxy mergers}

\subsubsection{Numerical models}

We use the same orbital parameters for all galaxy merger simulations. Orbital eccentricity is set to $e=1$, since N-body cosmological simulations of merging DMHs suggest that almost half of all orbits are close to parabolic, with $e=1 \pm 0.1$ ( Benson 2005; Khochfar \& Burkert 2006). The initial separation between galaxies is the sum of their virial radii, while pericentric distance is $R_{\text {peri }}=0.5 \% R_{\mathrm{vir}, 1}$, where $R_{\mathrm{vir}, 1}$ is the virial radius of the primary, i.e. more massive galaxy. Such a small pericentric distance leads to almost head-on collision and fast merging process that saves computational time.

SMBHs are added at the beginning of the merger simulations as massive particles, placed at the centre of mass of each progenitor galaxy. The centre of mass is calculated with respect to the most gravitationally bound 5 per cent bulge particles. Separation between galaxies during merger is calculated by tracing SMBH positions. We follow galaxy merger and the SMBH inspiral until separation between SMBHs drops below the gravitational softening length, i.e. below $0.1 \mathrm{kpc}$. Since final stages of SMBH mergers occur on sub-resolution scales, we adopt the the similar approach as Blecha et al. (2016), and add a time delay for SMBH mergers. The time delay depends on SMBH mass ratio $q$ as $0.1 \mathrm{Gyr} / \mathrm{q}$. Here we assume that SMBH mass ratio scales to the mass ratio of merging galaxies, i.e. $q=1$ for major mergers and $q=0.1$ for minor mergers. We follow galaxy mergers until two criteria are met: 1) separation between
SMBHs drops bellow $0.1 \mathrm{kpc}$, and 2) apocentric distance for further passages is $\lesssim 10 \mathrm{kpc}$. We assume that the SMBH merger is completed 0.1 Gyr after these criteria are met in the case of major mergers. Similarly, we add 1 Gyr time delay in the case of minor mergers.

Using Romulus 25 cosmological simulation Tremmel et al. (2018) investigated the distribution of time that components of SMBH binaries spend on separations $\lesssim 10 \mathrm{kpc}$ from each other. Their results suggest that most SMBH binaries will form close pairs in less than 1 Gyr, but that required time strongly depends on galaxy morphology and mass ratio of merging galaxies, where some galaxy mergers do not lead to $\mathrm{SMBH}$ pair formation. Close binary formation timescale is short for major mergers of massive galaxies, while after minor mergers, where satellite galaxy has low density stellar core, close pair will form several Gyr after the galaxy merger. This is consistent with our results. We note that in the simulation of minor merger remnant with mass $M_{\text {gal }}=10^{11} \mathrm{M}_{\odot}$ and $M_{\mathrm{BH}}-M_{\text {halo }} \mathrm{SMBH}, \mathrm{SMBH}$ merger may not be completed within 10 Gyr. Even though during pericentric passages distance between SMBHs drops below $0.1 \mathrm{kpc}$, apocentric distances are $\lesssim 20 \mathrm{kpc}$. It is unclear whether SMBHs in galaxies with the same orbital parameters would merge within a Hubble time, even in gas rich galaxies. Merger simulations of gas rich galaxies with the same mass ratio suggest that central SMBHs may never merge if their orbits have small first pericentric distance, as in our simulations. During the first pericentric passage strong tidal forces remove the gas from the satellite galaxy, and the central SMBH stays in the halo of the primary galaxy. At that separation, the estimated timescale for orbital sinking of the secondary SMBH may be longer than a Hubble time (Callegari et al. 2009, 2011). Despite this uncertainty we include this case in our analysis with assumption that SMBHs merge after separations between SMBHs drops below our softening length, and with a relaxed criterion that apocentric distance for further passages is $\lesssim 20 \mathrm{kpc}$.

\subsubsection{Analytical models}

Analytical merger remnant galaxies are modeled in the similar manner as the progenitor galaxies, with the same density profiles described in Section 2.1.2. We assume that major merger of two disc galaxies produces an elliptical galaxy, while disc component is conserved in minor mergers. We make numerical models of isolated galaxies and then fit their mass profiles in order to produce analytical galaxies with the same properties. Mass of each galaxy component is the sum 
of DM, stellar and gas masses in progenitor galaxies. Parameters of analytical post-merger galaxies are summarized in Table 2 .

We repeat the same procedure described in Section 2.2 in order to follow trajectories of the recoiling SMBHs in numerical and analytical merger remnant galaxies.

We note that our analytical merger remnant models are spherically symmetric, unlike numerical models which are not perfectly spherical. We perform test runs in order to investigate if deviation from spherical symmetry in numerical post-merger galaxies could contribute to the difference between models. Test runs follow a recoiling SMBH in a major merger remnant, since mergers of equal mass galaxies are expected to produce remnants with higher degree of asymmetry. However, changing direction of a SMBH kick from orthogonal to parallel with respect to the disc plane results in less than 1 per cent change in the escape velocity.

\section{RESULTS}

Fig. 4 shows galaxy escape velocity in analytical models as a function of escape velocity in numerical models for galaxies with mass of $M_{\text {gal }}=10^{12} \mathrm{M}_{\odot}$ (upper panel) and $M_{\text {gal }}=$ $10^{11} \mathrm{M}_{\odot}$ (lower panel). Triangles and squares represent major and minor merger remnants, respectively. Filled symbols show galaxy models with over-massive central SMBH and open symbols galaxies with a $\mathrm{SMBH}$ on $M_{\mathrm{BH}}-M_{\text {halo }}$ relation.

\subsection{Comparison between analytical models}

If different analytical models are compared with each other, Fig. 4 shows that galaxies with over-massive SMBHs $\left(M_{\mathrm{BH}}=M_{\text {gal }} / 10^{3}\right)$ have greater escape velocities than galaxies with $M_{\mathrm{BH}}-M_{\text {halo }} \operatorname{SMBHs}\left(M_{\mathrm{BH}}=M_{\text {gal }} / 10^{5}\right)$. This is the consequence of two effects: 1 ) galaxy models with overmassive SMBHs also have massive, extended bulges and thus greater density inside central $\sim 20 \mathrm{kpc}$ (mass profiles will be shown on Fig. 5), and 2) more massive SMBHs experience greater drag force due to the dynamical friction (equation (8)). For a given SMBH mass, major and minor merger remnants have approximately equal escape velocities. Even though major mergers will form an elliptical galaxy and a minor merger product is a disc galaxy, changes in the total mass profiles between analytical models are negligible.

\subsection{Comparison between numerical models}

In the numerical models, mergers lead to redistribution of mass within the merger remnant galaxy. During the interaction between two galaxies a part of gravitational potential energy is converted into the kinetic energy of individual particles and back. This energy conversion is described by the time dependent virial theorem:

$\frac{1}{2} \frac{\mathrm{d}^{2} I}{\mathrm{~d} t^{2}}=2 T+V$

where $T$ and $V$ are kinetic and potential energy of the galaxy, respectively, and $I$ is the moment of inertia tensor. Galaxy is virialized, i.e. at equilibrium for $\ddot{I}=0$ and
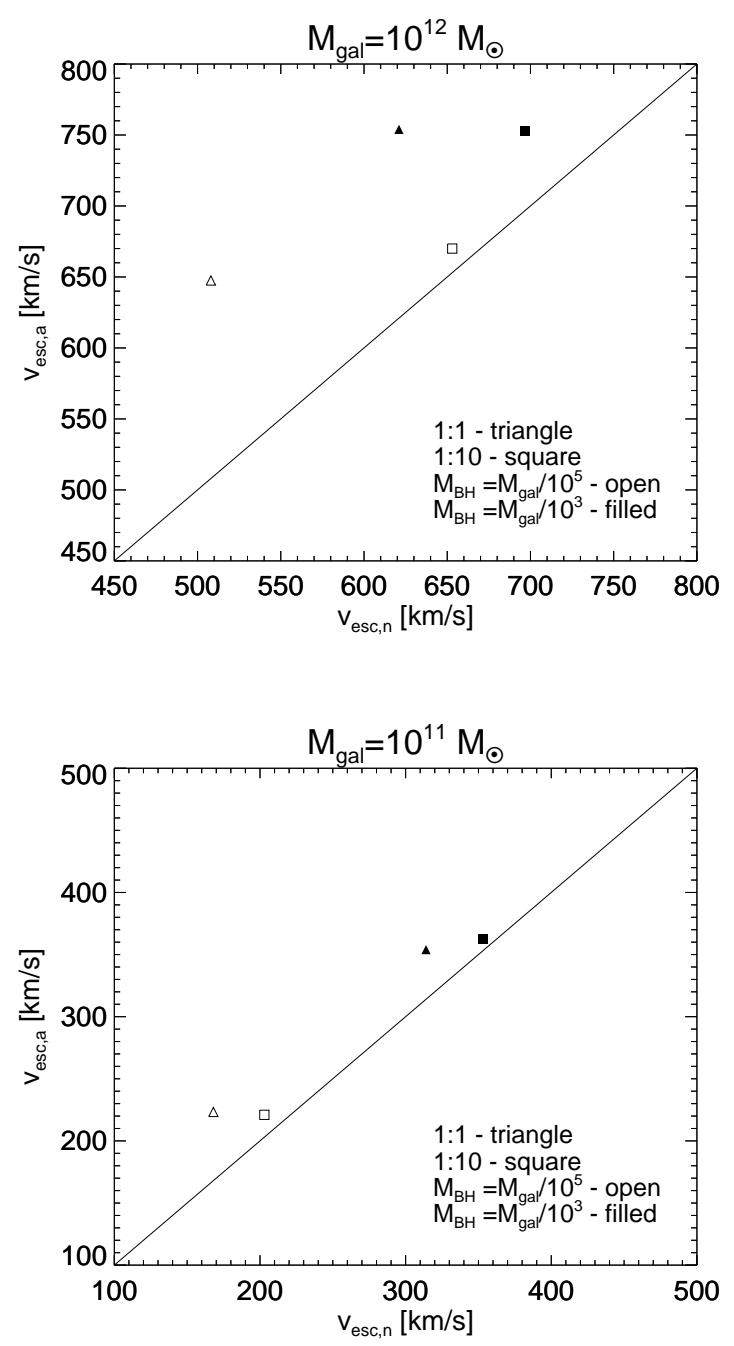

Figure 4. Escape velocities from galaxies in analytical models as a function of their escape velocities in numerical models, for galaxies with different central SMBH masses (open and filled symbols). Major merger remnants are represented with triangles and minor merger remnants with squares. We note that a relaxed criteria is imposed for minor merger of $M_{\text {gal }}=10^{11} \mathrm{M}_{\odot}$ galaxy with $M_{\mathrm{BH}}=M_{\mathrm{gal}} / 10^{5} \mathrm{SMBH}$ : we assume that $\mathrm{SMBH}$ merger is completed 1 Gyr after separation between SMBHs drops below 0.1 $\mathrm{kpc}$, while apocentric distance for further passages is $\lesssim 20 \mathrm{kpc}$ (we refer to Section 2.3.1 for more details)

$T=-E, V=2 E$, where $E$ is the total energy of the galaxy. During galaxy merger, the total energy does not change, however kinetic and potential energy will oscillate around the equilibrium values. In such systems energy of individual particles is not conserved. In the process called violent relaxation (Lynden-Bell 1967) energy of some particles will decrease and those particles will sink to the remnants centre, increasing its central density. On the other hand, energy of other particles will increase leading to the extended mass distribution. Weakly bound particles can escape the host galaxy potential, which results in mass lost during mergers. This process is especially efficient during major mergers. Importance of violent relaxation for major mergers is investigated by Hilz et al. (2012). The authors also showed that in 

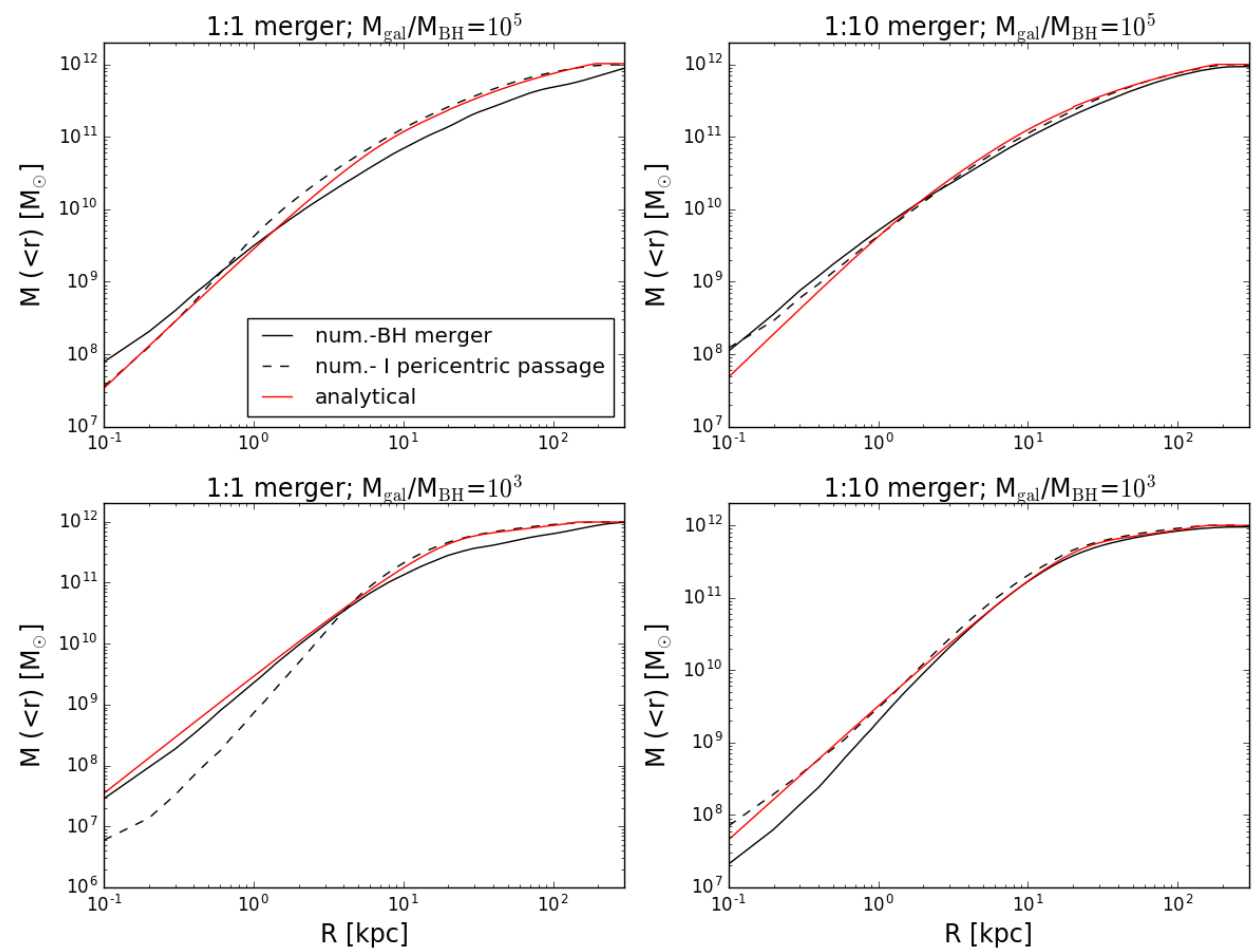

(a) Total mass profile of $10^{12} \mathrm{M}_{\odot}$ galaxies
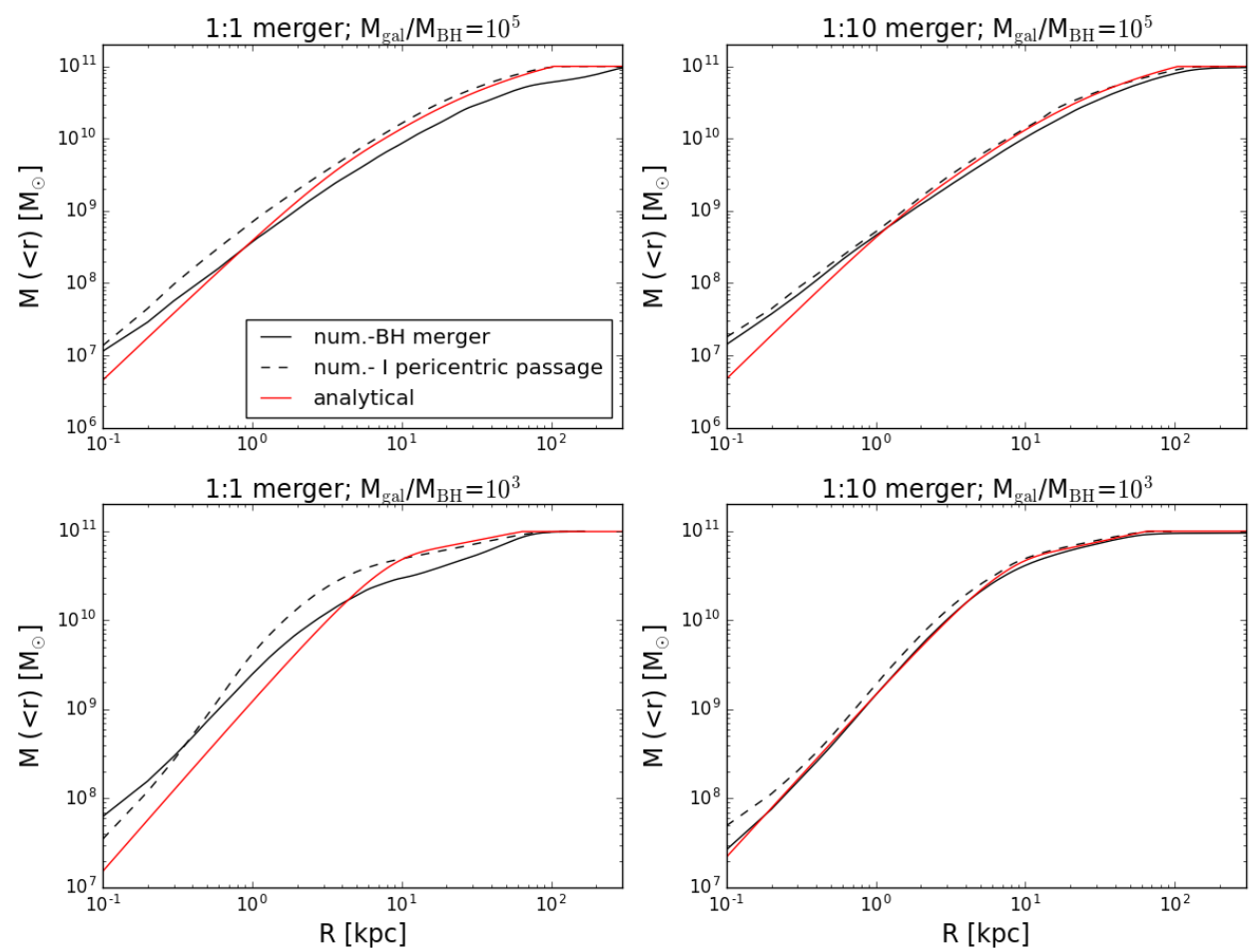

(b) Total mass profile of $10^{11} \mathrm{M}_{\odot}$ galaxies

Figure 5. Total mass profile of numerical and analytical models of merger remnant galaxies. Red lines correspond to the analytical models and black lines to numerical models at the first pericentric passage (dashed lines) and SMBH merger (solid lines). Panels show major and minor merger remnants of galaxies with different central SMBH masses. 

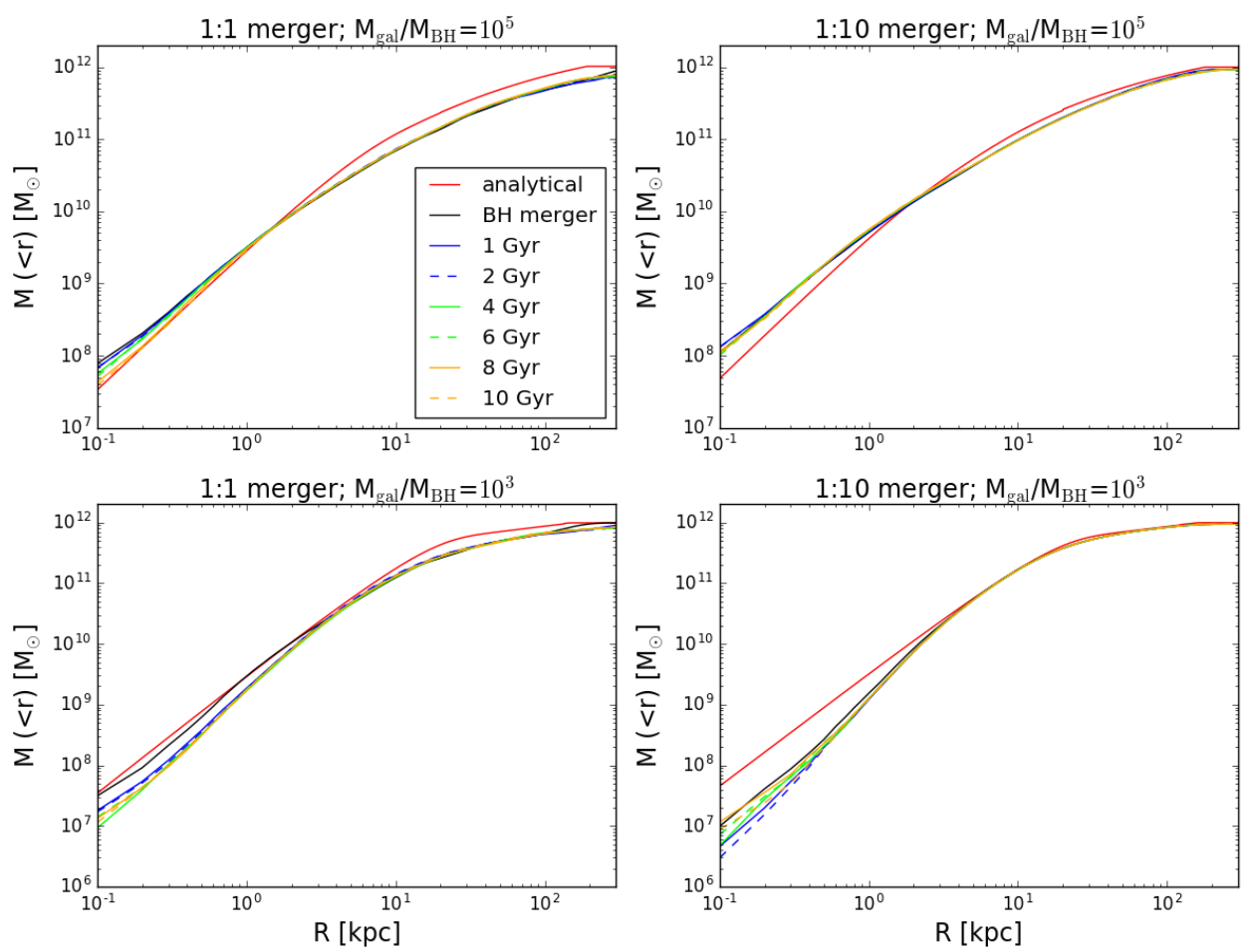

(a) Total mass profile of $10^{12} \mathrm{M}_{\odot}$ galaxies
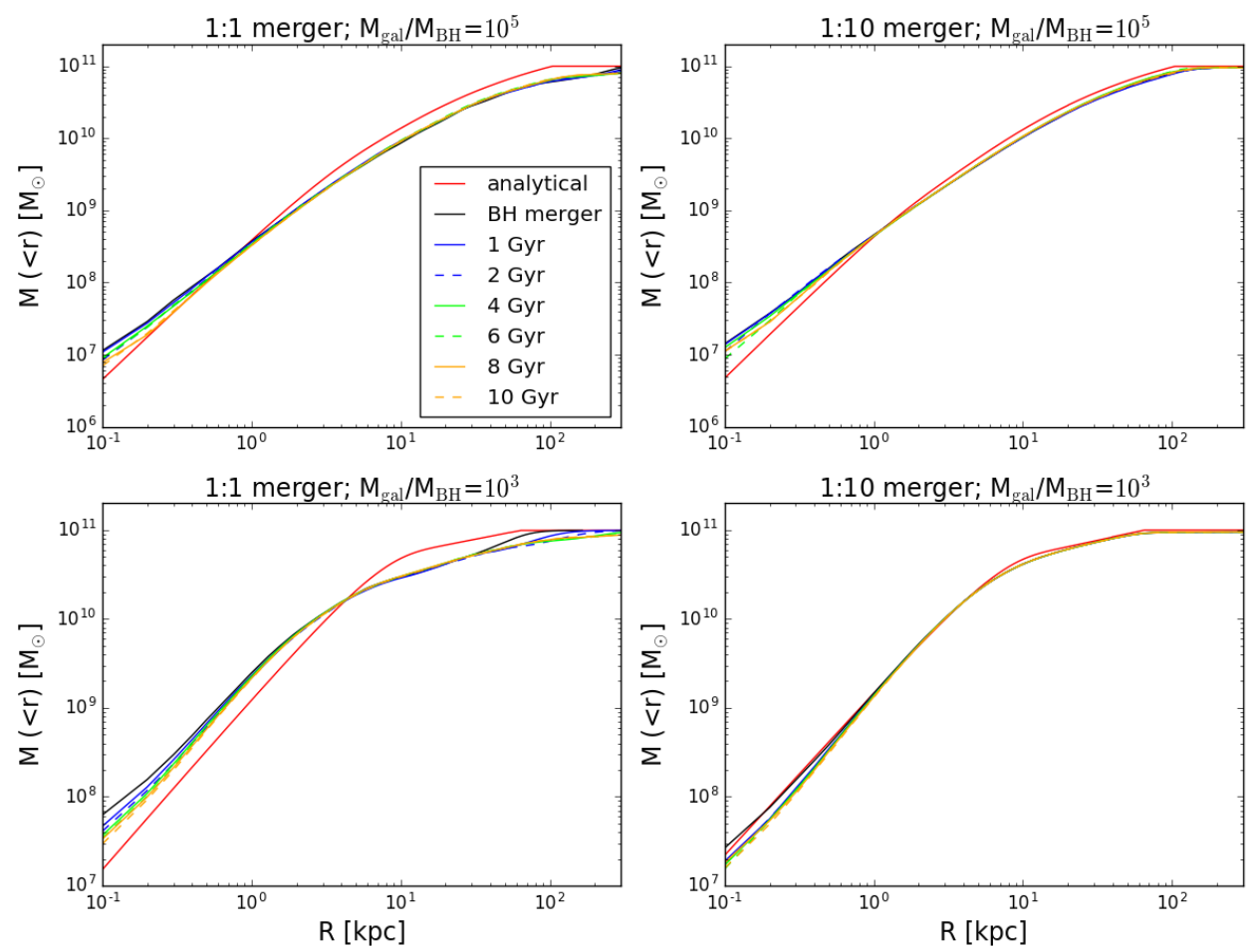

(b) Total mass profile of $10^{11} \mathrm{M}_{\odot}$ galaxies

Figure 6. Evolution of total mass profiles of numerical merger remnant galaxies. Black lines represent mass profile at the time of SMBH merger, while blue, green and orange lines show evolution of the total mass profiles during the simulation. Red lines represent total mass profile in analytical models. 
the case of minor mergers violent relaxation influences only particles from secondary galaxy and has no significant effect on the distribution of the particles in the primary galaxy.

Since SMBH ejection in numerical models occurs in merger remnants whose mass and potential are redistributed as a consequence of the recent galaxy interactions, the recoiling SMBH trajectory in numerical models is expected to differ from a trajectory of a SMBH moving through postmerger galaxy with stable, analytical potential.

When different numerical models are compared mutually, Fig. 4 shows that major merger remnants have lower escape velocities than galaxies formed in minor mergers. Major mergers lead to more significant mass redistribution, which is shown in Fig. 5. Under the influence of violent relaxation process during major mergers weakly bound particles are transfered beyond virial radius of the merger remnant. This results in extended mass distribution with lower mass profile. Escape velocities in weakened potential of major merger remnant are $\lesssim 25$ per cent lower compared to minor merger remnants. Over-massive SMBHs in numerical models also need larger kick velocities in order to leave their host centre, due to the presence of massive bulge, and greater influence of dynamical friction force. Changes in the galaxy mass profile induced by mergers will be discussed in Fig. 5 .

\subsection{Comparison between analytical and numerical models}

Finally, if analytical models are compared with numerical models, Fig. 4 shows that numerical galaxies have up to 25 per cent lower escape velocities. The greatest difference between analytical and numerical models is noticed when escape velocities from major merger remnants with a central SMBH on $M_{\mathrm{BH}}-M_{\text {halo }}$ relation are compared (open triangles on Fig. 4). Those differences are caused by violent relaxation process during major mergers in numerical models, which leads to the decrease of mass in the mass profiles of the numerical remnant galaxies.

Escape velocities from numerical 1:10 merger remnants are at most 8 per cent lower compared to the analytical models. Secondary galaxy will experience significant mass redistribution during minor merger. However, the satellite galaxy has only lesser contribution to the total potential of the merger remnant. Changes in the total mass profile caused by violent relaxation process during minor mergers are negligible compared to the changes during major mergers. This leads to the similar escape velocities in numerical and analytical minor merger models.

\subsection{Comparing mass profiles}

In order to further explain differences between escape velocities from different galaxy models, we calculate total mass profiles of merger remnants. Fig. 5 shows the cumulative mass enclosed within a given radius for different galaxy models. Major merger remnants are represented in the left panels and minor merger remnants in the right panels. Galaxies with $M_{\mathrm{BH}}-M_{\text {halo }} \mathrm{SMBH}$ are shown in the upper panels and galaxies with over-massive SMBH in the lower panels. Red solid lines correspond to analytical merger remnant models and black lines to numerical models. Total mass profile of numerical models is calculated from the snapshot taken at the time of the first pericentric passage (dashed lines) and at the time of SMBH merger (solid lines).

As discussed in Section 3.2 violent relaxation during major mergers causes bound particles to become more bound and sink to the remnants centre, while weakly bound particles become unbound and escape host galaxy (Lynden-Bell 1967). Fig. 5 shows that at the time of the first pericentric passage, total mass of merging galaxies makes a close fit to the analytical mass profile at radii greater than several $\mathrm{kpc}$ (black dashed lines). However, at the time of SMBH merger/ejection (black solid lines), potential of the major merger remnant has already evolved, and when compared to analytical, its mass profile is lower at large radii and higher at small radii. A fraction of particles is pushed beyond virial radius which flattens the mass profile, while some particles sink to the bottom of the hosts potential well, increasing central density. However, this central mass boost is not sufficient to suppress the SMBH recoil, leading to the lower escape velocities in numerical major merger remnants. Thus, the evolving numerical model is a more realistic description of dynamical processes in galaxies with merging SMBHs. Static analytical models used in literature, overestimate the SMBHs escape velocities, hence, underestimate the number of offset AGNs.

Minor mergers do not induce significant changes in mass profile of the remnant galaxy. Elliptical galaxies with overmassive SMBH show mass loss in central kpc. This mass loss is due to interactions of massive central SMBHs with particles in central region. At large radii deviations from analytical models are negligible.

Fig. 6 shows evolution of total mass profiles of numerical merger remnants over 10 Gyr. Similarly as in Fig. 5, different panels correspond to major and minor merger remnants of galaxies with different central SMBH masses. Black lines show total mass profiles of numerical galaxies at the time of SMBH merger, while blue, green and orange lines represent evolution of the post-merger galaxy during the simulation of SMBH ejection. For a comparison, red lines represent the total mass profile in analytical models. After the SMBH merger and the ejection of the newly formed SMBH, postmerger galaxies do not show significant evolution of mass profiles. Changes in mass profiles are constrained almost entirely to the central kpc of the merger remnant galaxy. Initial central mass boost induced by major mergers is followed by gradual decreasement of central mass profile during the simulation. At large radii mass profiles are not affected by this evolution.

\subsection{Recoiling SMBH trajectories}

Fig. 7 shows the maximal separation from a galaxy centre reached by recoiled SMBH on a bound orbit over a Hubble time, for numerical (upper panel) and analytical (lower panel) models. In both analytical and numerical models SMBHs with kick amplitudes $v_{\text {kick }} \lesssim 300 \mathrm{~km} \mathrm{~s}^{-1}$ for $10^{12} \mathrm{M}_{\odot}$ remnants and $v_{\text {kick }} \lesssim 100 \mathrm{~km} \mathrm{~s}^{-1}$ for $10^{11} \mathrm{M}_{\odot}$ remnants, stay within central $\sim 10 \mathrm{kpc}$. Only SMBHs that receive kick velocities close to the hosts escape velocity can reach separation of $r_{\max }>100 \mathrm{kpc}$. For these largest separations, Fig. 7 shows that SMBHs in numerical models reach them with kicks substantially smaller than in analytical models. 

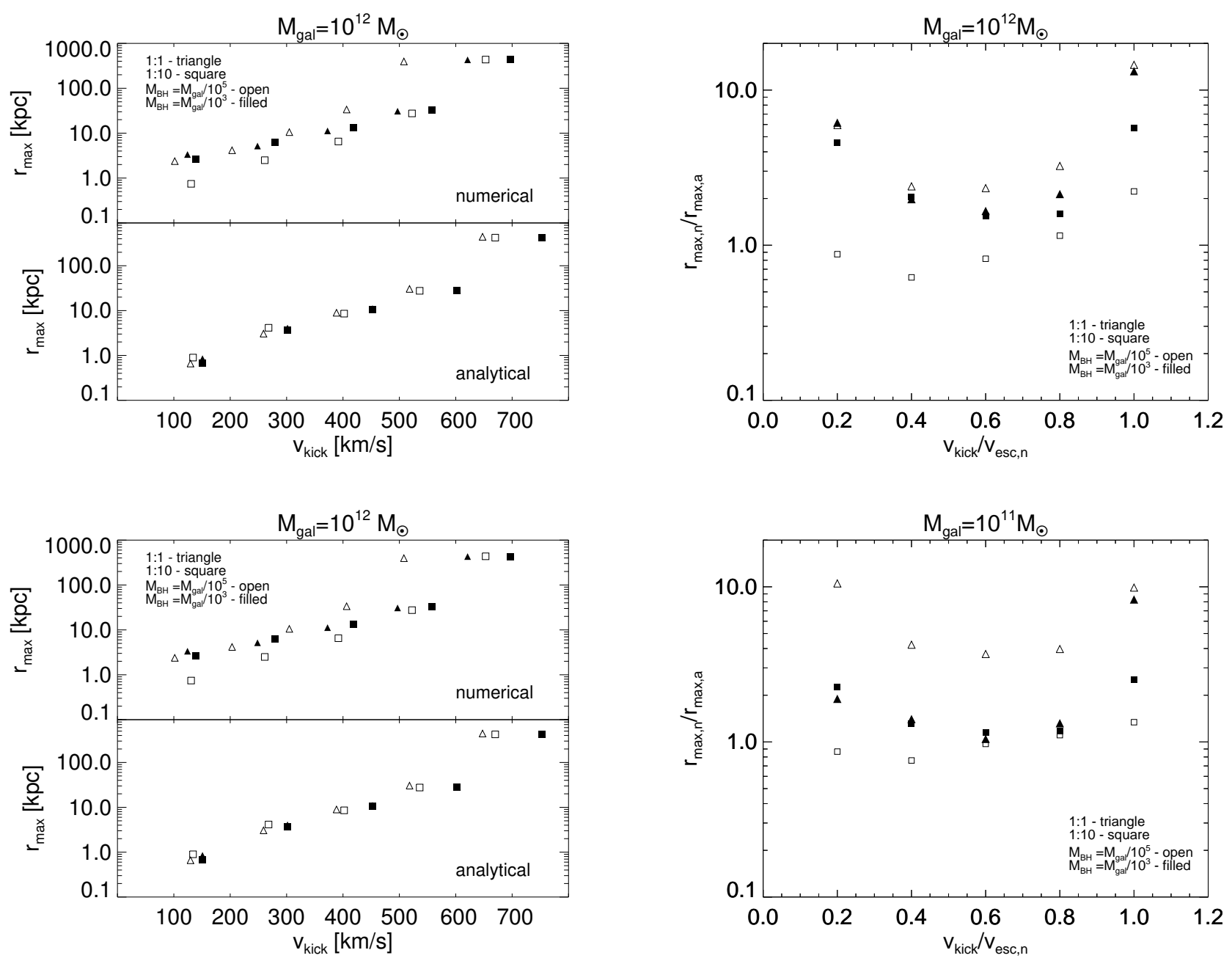

Figure 7. Maximal separation of a recoiling SMBH from the galaxy centre over a Hubble time, as a function of kick velocity. Kick velocities are chosen to represent $v_{\text {kick }}=$ $0.2,0.4,0.6,0.8,1.0 \times v_{\text {esc }}$ for each galaxy model.

For example, SMBH in numerical major merger remnant of $10^{12} \mathrm{M}_{\odot}$ galaxy whose kick amplitude is $v_{\text {kick }} \sim 500 \mathrm{~km} \mathrm{~s}^{-1}$ will reach approximately the same separation as SMBH with kick amplitude $v_{\text {kick }} \sim 650 \mathrm{~km} \mathrm{~s}^{-1}$ in analytical model (open triangles on Fig. 7).

In order to further investigate differences between numerical and analytical potentials, we assign kick velocities to recoiling SMBHs in both analytical and numerical merger remnants and compare their distances from galactic centres. In Fig. 8 we show ratio of the maximal separation from a galaxy centre reached by recoiled SMBH over a a Hubble time in those two models $\left(r_{\max , \mathrm{n}} / r_{\max , \mathrm{a}}\right)$ as a function of $v_{\text {kick }} / v_{\text {esc,n }}$. Here, $v_{\text {esc,n }}$ is the escape velocity in numerical post-merger galaxy. Recoiling SMBHs will reach greater galactocentric distance in numerical models, which is especially pronounced for major merger remnants. For low kick velocities, $v_{\text {kick }} / v_{\text {esc,n }}=0.2, \mathrm{SMBHs}$ in numerical models will reach several $\mathrm{kpc}$, while in analytical models SMBHs will stay within central kpc. For kick amplitudes equal to the numerical escape velocity, differences between models become even more significant. SMBHs in numerical major

Figure 8. Ratio of the maximal separation of a recoiling SMBH in numerical and analytical models as a function of $v_{\text {kick }} / v_{\text {esc,n }}$.

merger remnants reach distances up to 10 times larger than in the analytical models. This result can have large consequences for the predicted distributions of offest AGNs.

Further, we compare our results with the distribution of the ejected SMBHs in Illustris simulation (Blecha et al. 2016). Fig. 9 shows the maximal separation from a galaxy centre reached by recoiled SMBH over a Hubble time as a function of $v_{\text {kick }} / v_{\text {esc,n }}$ for our numerical models. Diamond symbols show galaxies with mass $M_{\text {gal }}=10^{12} \mathrm{M}_{\odot}$, while asterisk symbols represent galaxies with mass $M_{\text {gal }}=10^{11} \mathrm{M}_{\odot}$. Shaded region represents the range of maximal galactocentric separations of recoiled SMBHs calculated by Blecha et al. (2016). The authors assigned SMBH kick velocities calculated from a distribution that assumes random premerger spin orientation. Spin magnitude is chosen from a distribution that peaks at $a \sim 0.7$, where $a$ is dimensionless spin parameters taking values from $a=0$ for nonrotating SMBHs to $a \lesssim 1$ for SMBHs with maximal rotation. Distribution of SMBH kick amplitudes resulting from such spin magnitude and spin orientation distributions peaks at $v_{\text {kick }} \sim 300 \mathrm{~km} \mathrm{~s}^{-1}$ and has long tail toward low kick velocities, although high kicks are also possible. Fig. 9 shows that the maximal distances from the host centre that SMBHs 


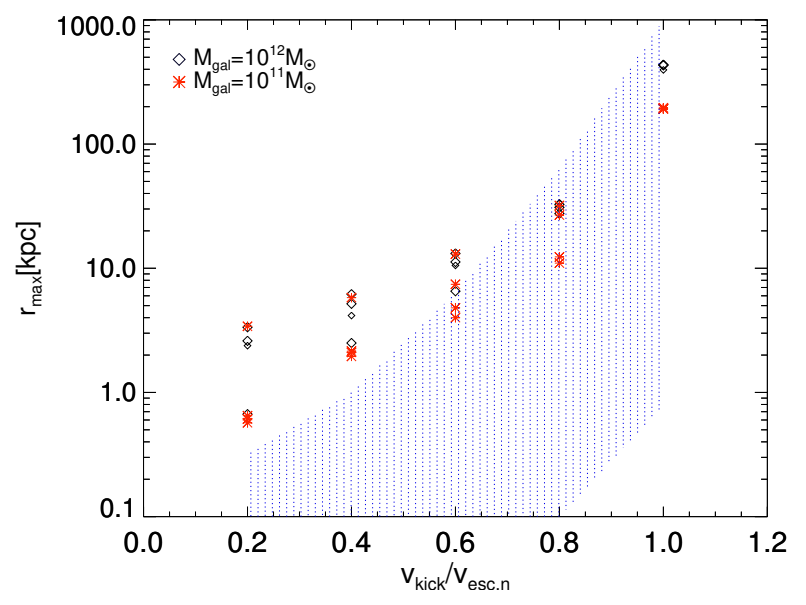

Figure 9. Maximal separation of a recoiling $\mathrm{SMBH}$ from the galaxy centre over a Hubble time as a function of $v_{\text {kick }} / v_{\text {esc,n }}$, for galaxies with mass $M_{\text {gal }}=10^{12} \mathrm{M}_{\odot}$ (diamond symbols) i $M_{\text {gal }}=10^{11} \mathrm{M}_{\odot}$ (asterisk symbols). Shaded region represents results obtained by Blecha et al. (2016).

could reach in our numerical models are greater compared to predictions made by Blecha et al. (2016) for kick velocities $v_{\text {kick }} / v_{\text {esc }, \mathrm{n}}<0.8$.

Blecha et al. (2016) have shown that, for a random premerger SMBH spin orientation, kick velocity distribution peaks at $v_{\text {kick }} / v_{\text {esc }} \sim 0.3$. For such kick amplitudes Blecha et al. (2016) model predicts that recoiling SMBHs will stay within central kpc, while in numerical models SMBHs can reach $\sim 10 \mathrm{kpc}$. SMBHs that reach greater galactocentric distance also spend more time on bound orbits outside of the the galactic nucleus, thus for the given $v_{\text {kick }} / v_{\text {esc }}$ ratio numerical models predict more spatially offset AGNs.

Additional difference between analytical and numerical models can be noticed if we compare escape velocities from the host galaxies with the expected kick velocity distribution for different SMBH spin models, calculated by Blecha et al. (2016) (their fig. 1). If the pre-merger SMBH spins are always aligned with the orbital angular momentum and with each other, kick velocities are always lower than escape velocities of $M_{\text {gal }}=10^{12} \mathrm{M}_{\odot}$ galaxies in analytical models. SMBH could occasionally leave analytical merger remnant with mass $M_{\text {gal }}=10^{11} \mathrm{M}_{\odot}$ and central $\mathrm{SMBH}$ on $M_{\mathrm{BH}}-M_{\text {halo }}$ relation. Similarly to our analytical results, Blecha et al. (2016) have found that SMBHs do not get kick velocities $v_{\text {kick }} / v_{\text {esc }}>0.8$ if SMBH alignment is efficient. In numerical models, SMBH kick velocities taken from the same distribution could be sufficient to eject SMBHs from $M_{\text {gal }}=10^{11} \mathrm{M}_{\odot}$ galaxies and from the major merger remnant of $M_{\text {gal }}=10^{12} \mathrm{M}_{\odot}$ galaxy with $M_{\mathrm{BH}}-M_{\text {halo }} \mathrm{SMBH}$. On the other side, if SMBH spins are close to their maximum value and randomly oriented prior to merger, SMBHs in both analytical and numerical models can get kicks $v_{\text {kick }} / v_{\text {esc }} \sim 1$.

Kick amplitude also depends on the merging SMBH mass ratio. Mergers of SMBHs with similar masses will possibly result with the highest kick amplitudes. Assuming that SMBH mass ratio scales to the mass ratio of merging galaxies, recoiling $\mathrm{SMBH}$ in the minor merger remnants of $M_{\text {gal }}=10^{12} \mathrm{M}_{\odot}$ galaxies may never receive kicks with amplitudes similar to the hosts escape velocity. Micic et al. (2011) have shown that the interval of possible kick velocities for SMBHs with mass ratio 0.1 is $50 \lesssim v_{\text {kick }} \lesssim 500 \mathrm{~km} \mathrm{~s}^{-1}$. In that case, SMBHs in minor merger remnants of massive galaxies will never get kicks $v_{\text {kick }} / v_{\text {esc }}>0.8$ in numerical models, or $v_{\text {kick }} / v_{\text {esc }}>0.7$ in analytical models.

Detections of offset AGNs would be helpful in constraining SMBH spin models, however observations of such events are challenging. There are several reasons for that: along with a large sample required for a detection and limited sensitivity or current instruments, offset AGN can be easily mistaken with other phenomena such as inspiral phase of SMBH mergers or Type IIn supernova. We refer to Blecha et al. (2019) for a recent overview of current searches for offset AGN.

Due to the lack of confirmed observations of recoiling SMBHs and offset AGN, our results cannot be directly compared with observations. Instead, results from large cosmological simulations can be used to make more concrete predictions about the number of offset AGNs in analytical and numerical models. This can be done semi-analytically by extracting major merger remnant galaxies with specific masses from the cosmological simulation. Those galaxies can be further used to calculate probability distributions of the recoiling $\mathrm{SMBH}$ positions with the respect to galaxy centre, for both analytical and numerical models. Using Monte Carlo method, different kick velocities resulting from different SMBH spin distributions, can be assigned to SMBHs from the simulation. The final position of the recoiling SMBH can be then calculated using predictions from analytical and numerical models presented in this paper. This would yield statistically relevant differences between ejected SMBHs in numerical and analytical models. However, such analysis is beyond the scope of this paper and it will be the subject of the following research.

\section{DISCUSSION AND CONCLUSIONS}

We have investigated trajectories of recoiling SMBHs in various analytical and numerical models of merger remnant galaxies whose components are $\mathrm{DMH}$, disc and bulge. Considered galaxies have masses of $M_{\text {gal }}=10^{12} \mathrm{M}_{\odot}$ and $M_{\text {gal }}=10^{11} \mathrm{M}_{\odot}$ and they host a central SMBH with mass $M_{\mathrm{BH}}=M_{\mathrm{gal}} / 10^{5}\left(M_{\mathrm{BH}}-M_{\text {halo }} \mathrm{SMBH}\right)$ or $M_{\mathrm{BH}}=M_{\mathrm{gal}} / 10^{3}$ (over-massive $\mathrm{SMBH}$ ). Mass of each component is calculated using scaling relations for a given mass of the central SMBH and the total galaxy mass. We separately model major and minor galaxy mergers in order to investigate if SMBH trajectories are sensitive to the mass ratio of the progenitor galaxies.

Previous studies of recoiling SMBHs predominantly used static analytical models of merger remnant galaxies in order to estimate SMBH escape velocities and the number of offset AGNs. Our goal was to extend those analytical models of merger remnants and construct more realistic numerical counterparts in order to compare possible differences in recoiling SMBH trajectories. First we have shown that analytical and numerical models of isolated progenitor galaxies produce the same results for the escape velocity and the maxi- 
mal separation the SMBH could reach over a Hubble time. Next, we simulate merger of progenitor galaxies and show that SMBH trajectories in numerical remnants differ from those in analytical models. Initial conditions for numerical galaxy models are generated using Galact ICs code (Kuijken \& Dubinski 1995; Widrow \& Dubinski 2005; Widrow, Pym \& Dubinski 2008), while galaxy merger simulations are carried out using GADGET-2 code (Springel 2005).

In our model galaxy escape velocity is defined as a SMBH kick velocity necessary for a SMBH to return to its host halo after $\gtrsim 10$ Gyr. We have shown that galaxies with equal total masses have different escape velocities due to different mass distributions resulting from the galaxy merger. Our main results are following:

(i) Static analytical models overestimate $\mathrm{SMBH}$ escape velocities compared to the evolving numerical models which represent a more realistic description of post-merger galaxies. Escape velocities in numerical models are up to $\sim 25$ per cent lower compared to analytical models. This difference arises from redistribution of mass within post-merger galaxy. During major mergers violent relaxation process causes bound particles to become more bound and sink to the remnant centre, and weakly bound particles to become unbound and escape the host galaxy potential. Escaping particles will make shallower mass profile at large radii and reduce escape velocity in major merger remnants. During minor mergers mass redistribution is not efficient enough to change the primary galaxy potential and only lesser changes in remnants escape velocities are noticed.

(ii) In agreement with the previous point, mass ratio of the progenitor galaxies also influences escape velocities of $\mathrm{SMBH}$ in post merger hosts. In numerical models, $\mathrm{SMBH}$ escape velocities from major merger remnants are up to $\sim 25$ per cent lower than escape velocities from numerical minor merger remnants, for the given SMBH mass. In analytical models escape velocities from major and minor merger remnants are approximately equal since analytical models do not account for mass redistribution induced by violent relaxation process during galaxy mergers.

(iii) Decreasing modeled SMBH mass from $M_{\mathrm{BH}}=$ $M_{\text {gal }} / 10^{3}$ to $M_{\mathrm{BH}}=M_{\text {gal }} / 10^{5}$ results in $\lesssim 45$ per cent lower escape velocities in both analytical and numerical models. This occurs because galaxies that harbor massive central SMBHs also have massive bulges $\left(M_{*}-M_{\mathrm{BH}}\right.$ relation) which suppress SMBHs recoils. Additional increasement in escape velocities is due to greater gravitational drag force experienced by over-massive SMBHs (equation (8)).

(iv) Our model suggests that offset AGNs are more common in numerical models of major merger remnants. Kick velocities needed to remove a SMBH from the galaxy centre are lower, which would result in the greater possibility of offset AGNs detection, regardless of the SMBH spin distribution models. Differences between numerical and analytical models are significant for both low and hight kick amplitudes. For $v_{\text {kick }} / v_{\text {esc, n }}=0.2$, SMBHs in analytical models will stay inside central $\mathrm{kpc}$, while in numerical models SMBHs can reach several kpc. Greater separations are both easier to detect and the return time of a recoiling SMBH is longer. For $v_{\text {kick }} / v_{\text {esc }, \mathrm{n}} \sim 1$, SMBHs in numerical major merger remnants can reach several hundreds of $\mathrm{kpc}$, while in analytical models maximal separation from the host cen- tre in several tens of kpc. Additionally, the ejected SMBH also carries an accretion disc whose mass decreases as $v_{\text {kick }}^{2}$ (Blecha \& Loeb 2008), so the accretion timescale is longer for SMBHs with lower kick velocities, which in turn increases possibility of detection.

Escape velocities from galaxies with mass of $M_{\text {gal }}=$ $10^{12} \mathrm{M}_{\odot}$ in numerical models are in the range $v_{\text {esc }} \sim$ $500-700 \mathrm{~km} \mathrm{~s}^{-1}$, depending on the SMBH mass and the formation channel (minor or major mergers). If pre-merger SMBH spins are aligned with the orbital angular momentum and with each other only rare SMBHs can have kick amplitudes large enough to permanently leave major merger remnant with a central SMBH on $M_{\mathrm{BH}}-M_{\text {halo }}$ relation. SMBHs in other models are not expected to get kick velocities $v_{\text {kick }} / v_{\text {esc }}>0.8$. On the other side, if SMBH spins are randomly oriented even SMBHs with mass $10^{9} \mathrm{M}_{\odot}$ could be occasionally ejected from their host centre. Escape velocities from galaxies with mass of $M_{\text {gal }}=10^{11} \mathrm{M}_{\odot}$ are lower, $v_{\text {esc }} \sim 170-350 \mathrm{~km} \mathrm{~s}^{-1}$ and greater number of SMBHs are expected to be found outside their hosts, regardless of $\mathrm{SMBH}$ spin parameter and its orientation. Our numerical models also show that galaxies formed by major mergers have relatively low escape velocities which could negatively influence merger driven SMBH growth, since major mergers can trigger episodes of gas accretion and exponential SMBH growth.

As discussed above, more concrete and statistically relevant comparison between numerical and analytical models would be to calculate probability distributions for SMBH positions in numerical and analytical models, using major merger remnants extracted from a cosmological simulations. This calculation will be the subject of the following research.

\section{ACKNOWLEDGEMENTS}

This work was supported by the Ministry of Education, Science and Technological Development of the Republic of Serbia through project no. 176021, "Visible and Invisible Matter in Nearby Galaxies: Theory and Observations. We thank the anonymous referee for thoughtful comments that improved our paper.

\section{REFERENCES}

Begelman M. C., Blandford R. D., Rees M. J., 1980, Nature, 287, 307

Benson A. J., 2005, MNRAS, 358, 551

Berczik P., Merritt D., Spurzem R., Bischof H.P., 2006, ApJ, 642, L21

Berentzen I., Preto M., Berczik P., Merritt D., Spurzem R., 2009, ApJ, 695, 455

Binney J., Tremaine S., 2008, Galactic Dynamics: Second Edition

Blecha L., Loeb A., 2008, MNRAS, 390, 1311

Blecha L., Cox T. J., Loeb A., Hernquist L., 2011, MNRAS, 412, 2154

Blecha L., Sijacki D., Kelley L. Z., Torrey P., Vogelsberger M., Nelson D., Springel V., Snyder G., Hernquist L., 2016, MNRAS, 456, 961

Blecha L., Brisken W., Burke-Spolaor S., Civano F., Comerford 
J., Darling J., Lazio T. J. W., Maccarone T. J., 2019, Science White Paper submitted to the Astro2020 Decadal Survey, arXiv:1903.09301

Bovy J., Rix H.-W., 2013, ApJ, 779, 115

Bovy J., 2015, ApJS, 216, 29

van den Bosch F. C., Jiang F., Hearin A., Campbell D., Watson D., Padmanabhan N., 2014, MNRAS, 445, 1713

Callegari S., Mayer L., Kazantzidis S., Colpi M., Governato F., Quinn T., Wadsley J., 2009, ApJ, 696, L89

Callegari S., Kazantzidis S., Mayer L., Colpi M., Bellovary J. M., Quinn T., Wadsley J., 2011, ApJ, 729, 85

Campanelli M., Lousto C. O., Zlochower Y., Merritt D., 2007, Phys. Rev. Lett., 98, 231102

Chandrasekhar S., 1943, ApJ, 97, 255

Choksi N., Behroozi P., Volonteri M., Schneider R., Ma C. P., Silk J., Moster B., 2017, MNRAS, 472, 1526

Cox T. J., Jonsson P., Somerville R. S. Primack J. R., Dekel A.,2008, MNRAS, 384, 386

Dehnen W., 2001, MNRAS, 324, 273

Devecchi B., Rasia E., Dotti M., Volonteri M., Colpi M., 2009, MNRAS, 394, 633

Ebisuzaki T., Makino J., Okumura S. K., 1991, Nature, 354, 212

Escala A., Larson R. B., Coppi P. S., Mardones D., 2004, ApJ, 607,765

Escala A., Larson R. B., Coppi P. S., Mardones D., 2005, ApJ, 630,152

Ferrarese L., 2002, ApJ, 578, 90

Fiacconi D., Mayer L., Roškar R., Colpi M., 2013, ApJ, 777, L14

Gajda G., ÅĄokas E. L., Athanassoula E.. 2018, ApJ, 868, 10

Geehan J. J., Fardal M. A., Babul A., Guhathakurt P., 2006, MNRAS, 366, 996

Goicovic F. G., Sesana A., Cuadra J., Stasyszyn F., 2017, MNRAS, 472, 514

González J. A., Hannam M., Sperhake U., Brugmann B., Husa S., 2007, Phys. Rev. Lett., 98w1101G

Gualandris A., Merritt D., 2008, ApJ, 678, 780

Gualandris A., Read J., Dehnen W., Bortolas E., 2017, MNRAS, 464, 11

Guedes J., Madau P., Mayer L., Callegari S., 2011, ApJ, 729, 125

Haiman Z., 2004, ApJ, 613, 36.

Hilz M., Naab T., Ostriker J. P., Thomas J., Burkert A., Jesseit R., 2012, MNRAS, 3136, 311

Khan F. M., Just A., Merritt D., 2011, ApJ, 732, 89

Khan F. M., Holley-Bockelmann K., Berczik P., Just A., 2013, ApJ, 773, 100

Khan F. M., Fiacconi D., Mayer L., Berczik P., Just A., 2016, ApJ, 828, 73

Khochfar S., Burkert A., 2006, A\&A, 445, 403

Komossa S., Merritt D., 2008, ApJ, 689, L89

Kormendy J., Ho L. C., 2013, ARA\&A, 51, 511

Kuijken K., Dubinski J., 1995, MNRAS, 277, 1341

Loeb A., 2007, Phys. Rev. Lett., 99, 041103

Lousto C. O., Zlochower Y., 2011, Phys. Rev. Lett., 107, 1102

Lynden-Bell D., 1967, MNRAS, 136, 101

Madau P., Quataert E., 2004, ApJ, 606, L17

Marconi A., Hunt L. K., 2003, ApJ, 589, L21

Mayer L., 2013, Class. Quantum Gravity, 30, 244008

Mayer L., Kazantzidis S., Madau P., Colpi M., Quinn T., Wadsley J., 2007, Science, 316, 187

McConnell N. J., Ma C.-P., 2013, ApJ, 764, 184

McMillan P., 2011, MNRAS, 414, 2446

Merritt D., 1996, AJ, 111, 2462

Merritt D., Milosavljević M., Favata M., Hughes S.A., Holz D.E., 2004, ApJ, 607, L9

Micic M., Abel T., Sigurdsson S., 2006, MNRAS, 372, 154

Micic M., Holley Bockelmann K., Sigurdsson S., 2011, MNRAS, 414, 1127
Milosavljević M., Merritt D., 2001, ApJ, 563, 34

Moster B. P., Naab T., White S. D. M., 2013, MNRAS, 428, 3121

Navarro J. F., Frenk C. S., White S. D. M., 1997, ApJ, 490, 493

Power C., Navarro J. F., Jenkins A., Frenk C. S., White S. D. M., Springel V., Stadel J., Quinn T., 2003, MNRAS, 338, 14

Preto M., Berentzen I., Berczik P., Spurzem R., 2011, ApJ, 732, L26

Rantala A., Pihajoki P., Johansson P., Naab T., Lahén N., Sawala T., 2017, ApJ, 840, 53

Redmount I. H., Rees M. J., 1989, Comments on Astrophysics, 14,165

Roškar R., Mayer L., Fiacconi D., Kazantzidis S., Quinn T. R., Wadsley J., 2015, MNRAS, 449, 494

Schechter P. L., 2016, in IAU Symposium, Vol. 317, The General Assembly of Galaxy Halos: Structure, Origin and Evolution, ed. A. Bragaglia, M. Arnaboldi, M. Rejkuba, D. Romano, 3538

Schnittman J. D., 2007, ApJ, 667, L133

Sesana A., 2007, MNRAS, 382, L6

Sijacki D., Springel V., Haehnelt M. G., 2011, MNRAS, 414, 3656

Smole M., 2015, Serb. Astron. J, 191, 17

Springel V., 2005, MNRAS, 364, 1105

Tanaka T., Haiman Z., 2009, ApJ, 696, 1798

Tremmel M., Governato F., Volonteri M., Quinn T. R., Pontzen A., 2018, MNRAS, 475, 4967

Vogelsberger M., Genel S., Springel V., Torrey P., Sijacki D., Xu D., Snyder G., Bird S., Nelson D., Hernquist L., 2014a, Nature, 509, 177

Vogelsberger M., Genel S., Springel V., Torrey P., Sijacki D., Xu D., Snyder G., Nelson D., Hernquist L., 2014b, MNRAS, 444, 1518

Volonteri M., 2007, ApJ, 663, L5

Volonteri M., Gultekin K., Dotti M., 2010, MNRAS, 404, 2143

Widrow L. M., Dubinski J., 2005, ApJ, 631, 838

Widrow L. M., Pym B., Dubinski J., 2008, ApJ, 679, 1239

del Valle L., Escala A., Maureira-Fredes C., Molina J., Cuadra J., Amaro-Seoane P., 2015, ApJ, 811, 5

Zahid H. J., Geller M. J., Fabricant D. G., Hwang H. S., 2016, ApJ, 832, 20

Zahid H. J., Sohn J., Geller M. J., 2018, ApJ, 859, 96

Zhang T., Liao S., Li M., Gao L., 2019, MNRAS, 487, 1227

\section{APPENDIX A: MASS PROFILES OF PROGENITOR GALAXIES}

This paper has been typeset from a TEX/LATEX file prepared by the author. 

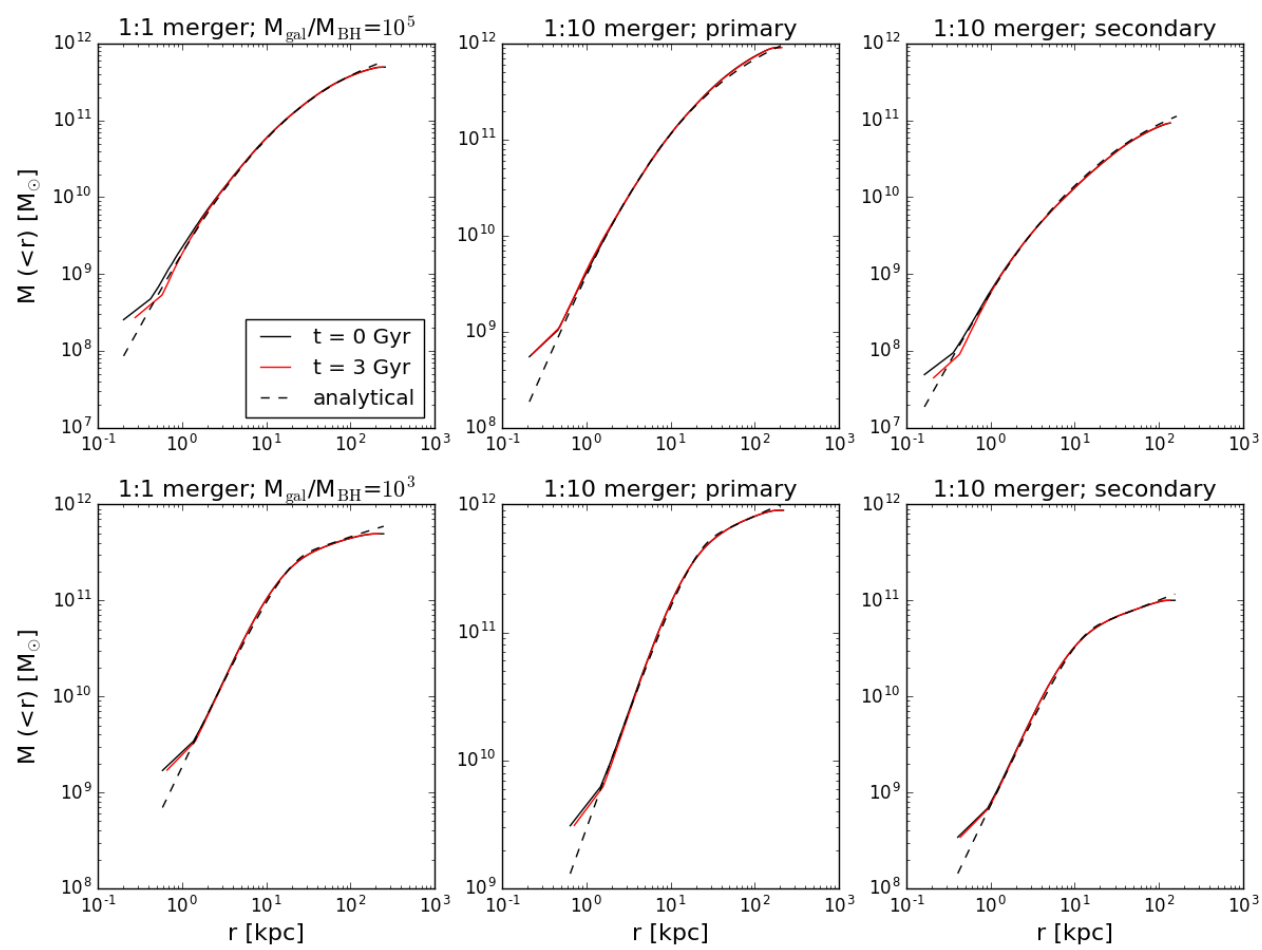

(a) Progenitors of $10^{12} \mathrm{M}_{\odot}$ galaxies
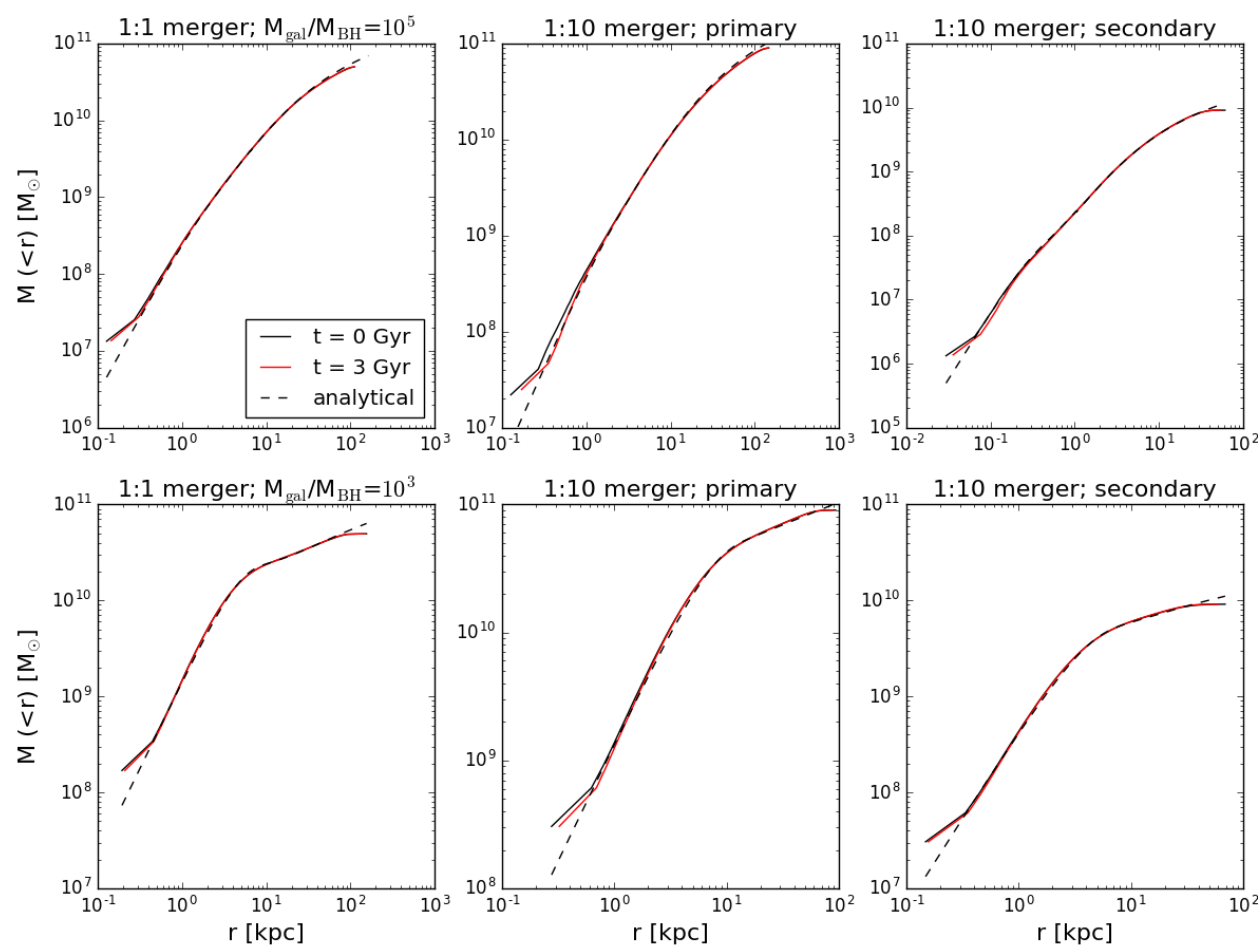

(b) Progenitors of $10^{11} \mathrm{M}_{\odot}$ galaxies

Figure A1. Total mass profiles in analytical (dashed lines) and numerical (solid lines) galaxy models. For numerical models mass profiles of individual galaxy components are shown at the beginning of the simulation and 3 Gyr later. Upper panels represent galaxies with SMBHs on $M_{\mathrm{BH}}-M_{\text {halo }}$ relation and lower panels galaxies with over-massive BHs. 